\title{
THE CRETACEOUS/TERTIARY BOUNDARY MASS EXTINCTION IN PLANKTIC FORAMINIFERA AT AGOST, SPAIN
}

\section{L'EXTINCTION EN MASSE DES FORAMINIFÈRES PLANCTONIQUES À LA LIMITE CRÉTACÉ/TERTIAIRE À AGOST, ESPAGNE}

\author{
by Eustoquio MOLINA*, Ignacio ARENILLAS* and José A. ARZ*
}

\begin{abstract}
The planktic foraminiferal mass extinction across the Cretaceous/Tertiary boundary at Agost (Spain) occurred over an extended period, with 7 species disappearing in the late Maastrichtian, 47 species extinct at the K/T boundary and 16 ranging into the earliest Danian. The species that became extinct at the $\mathrm{K} / \mathrm{T}$ boundary are large, complex tropical and subtropical forms dwelling in deep and intermediate water depths, which constitute only about $20 \%$ of the individuals in the population larger than 63 microns. Nevertheless, their disappearance constitutes the major and sudden extinction event in the history of planktic foraminifera. However, the small cosmopolitan surface dwellers with simple morphologies appear to survive and the last of them gradually disappear in the lower part of the $P$. pseudobulloides Biozone. This planktic foraminiferal extinction model can be interpreted as a catastrophic mass extinction that centred at the K/T boundary, and was superimposed on a gradual mass extinction which began in the late Maastrichtian and continued into the early Danian. The catastrophic pattern of extinction at the K/T boundary is very compatible with the effect of a large meteorite impact, whereas the gradual and extended pattern of extinction across the Maastrichtian-Danian transition is compatible with temperature and sea level changes that may be related to massive volcanism.
\end{abstract}

RÉSUMÉ. - L'extinction en masse des foraminifères planctoniques à travers la limite Crétacé/Tertiaire à Agost (Espagne) s'est produite au cours d'une certaine période; 7 espèces disparaissant au Maastrichtien supérieur, 47 à la limite $\mathrm{K} / \mathrm{T}$ et 16 au Danien inférieur. Les espèces ayant disparu à la limite $\mathrm{K} / \mathrm{T}$ sont grandes et complexes, des formes vivant en milieux océaniques profonds tropicaux et subtropicaux, qui ne constituent qu'environ $20 \%$ des individus de la population de taille supérieure à 63 microns. Cependant, leur disparition représente l'évènement d'extinction le plus important et soudain de l'histoire des foraminifères planctoniques. Toutefois, les petites formes cosmopolites ayant une morphologie simple et vivant dans les eaux superficielles semblent avoir survécu et la plupart ont disparu progressivement dans la partie inférieure de la Biozone à $P$. pseudobulloides. Ce modèle d'extinction des foraminifères planctoniques peut être considéré comme une extinction en masse catastrophique concentrée à la limite K/T et superposée à une extinction en masse graduelle qui avait commencé au Maastrichtien supérieur et continua jusqu'au Danien inférieur. Le modèle d'extinction catastrophique à la limite $\mathrm{K} / \mathrm{T}$ est bien compatible avec les effets de l'impact d'un grand météorite, tandis que le patron d'extinction graduelle au cours de la transition Mastrichtien-Danien l'est avec les changements de température et du niveau de la mer qui pourraient être en rapport avec un volcanisme massif.

Key-words : Cretaceous - Tertiary - Extinction - Biostratigraphy - Foraminifera - Betics - Spain.

Mots-clés : Crétacé - Tertiaire - Extinction - Biostratigraphie - Foraminifères - Bétiques - Espagne.

\section{INTRODUCTION}

The Cretaceous/Tertiary boundary (K/T) mass extinction model in planktic foraminifera and its interpretation has been a very controversial topic. The origin of this controversy can be found in the data that Smit presented at the Snowbird I Conference in 1980 , showing that all but one Cretaceous species suddenly became extinct at the K/T boundary, which he interpreted as the result of a large meteorite impact (Smit and Hertogen, 1980; Smit, 1982). These data were not questioned until Keller (1988) provided evidence that species extinctions extended across the $\mathrm{K} / \mathrm{T}$ boundary with about $1 / 3$ of the species surviving well into the Tertiary (Keller, $1988,1989 \mathrm{a}, \mathrm{b})$. 
The oldest detailed studies were published by Luterbacher and Premoli Silva (1964), who established the Globigerina eugubina Biozone at the $\mathrm{K} / \mathrm{T}$ boundary in Italy. The assemblage of this biozone was later recorded from the South Atlantic, Northwest Pacific, Gulf of Mexico and Caribbean deep-sea cores, which led Premoli Silva (1977) to conclude that the $G$. eugubina Biozone fauna is cosmopolitan, and represents the earliest Tertiary assemblage from which all Paleogene genera could be derived either directly or by inference. Subsequently, high resolution sampling of the K/T boundary at Caravaca (Spain) by Smit (1977, 1979 ) let to the discovery of a still earlier Tertiary planktic foraminiferal association below the $G$. eugubina Biozone. By this time Alvarez et al. (1979, 1980 ) proposed the now popular hypothesis that an extraterrestrial bolide caused the Cretaceous-Tertiary mass extinction. This has led to a tremendous interest in the $\mathrm{K} / \mathrm{T}$ mass extinction across disciplines ranging from paleontology to chemistry and astrophysies. Many authors have since tried to elucidate the model of extinction and their causes but few have studied continuous sections or at the necessary high sample resolution. Consequently, as a result of large sample spacing or hiatuses, mainly based on the Italian sections, it was assumed that Cretaceous planktic foraminifera suffered near total extinction precisely at the $\mathrm{K} / \mathrm{T}$ boundary.

During the late 1980 's, however, some workers studied more complete sections and sampled the critical intervals at the centimetre scale. As a result, they discovered a more gradual mass extinction pattern in planktic foraminifera (Keller, 1988, 1989 a, b, 1993 ; Canudo et al., 1991). Nevertheless, studying the same sections Smit (1990) has maintained his interpretation of a catastrophic pattern with all but one Cretaceous species (Guembelitria cretacea) extinct precisely at the $\mathrm{K} / \mathrm{T}$ boundary. To resolve this catastrophic versus gradual mass extinction controversy, a group of scientists re-sampled the El Kef section in Tunisia and samples were distributed for a "blind sample test". For this blind test six unlabeled samples were studied by four investigators, the result was exhibited at the Snowbird III Conference and both authors claimed that the data support their interpretations (Smit, 1994; Keller et al., 1995).

In order to elucidate this controversy we have studied the best section in Spain, located at Agost, which is very expanded and continuous, and well exposed across the K/T boundary. The planktic foraminifera are quite well preserved, the assemblages are rich and diverse, and there is almost no evidence of reworking. Thus, this excellent K/T boundary section provides a good opportunity to test the extinction model of Cretaceous species and the evolution of Tertiary species.

\section{MATERIALS AND METHODS}

The town of Agost is located in the Betic Cordillera of southeaster Spain and the section is placed about $1 \mathrm{~km}$ Northeast of the town (see detailed map in Canudo et al., 1991). The K/T boundary transition was sampled in a roadcut near the $13 \mathrm{~km}$ marker post and the Maastrichtian sequence closer to the town in a small gully opposite to the cemetery. Maastrichtian sediments consist of intercalated grey marls and marly limestone beds. The $\mathrm{K} / \mathrm{T}$ boundary is marked by a sharp contact between Maastrichtian grey marls and a thin $10 \mathrm{~cm}$ black clay layer with a basal $2 \mathrm{~mm}$ thin rust-red layer. This red layer contains the Ir anomaly and other impact evidence such as the Ni-rich spinels. The Tertiary sediments consist of marls and marly limestones that are of gray colour in the basal Paleocene and red at the top of the section.

All the samples were taken in a well exposed outcrop, but the outcrop was trenched across the $\mathrm{K} / \mathrm{T}$ boundary to remove surface contamination and obtain fresh unweathered bedrock. Samples were collected at cm-intervals across the critical $\mathrm{K} / \mathrm{T}$ boundary interval and at m-intervals below and above the boundary. A total of 68 samples were analyzed for this study. Samples were disaggregated in tap water and diluted $\mathrm{H}_{2} \mathrm{O}_{2}$, then washed through a 63 micron sieve and dried at $50^{\circ} \mathrm{C}$. Preservation of planktic foraminifera is good although original calcite shells are recrystalized.

The quantitative planktic foraminiferal analysis was based on representative splits (using a modified Otto microspliter) of 300 or more specimens in the size fraction larger than 63 microns. All the representative specimens were picked, identified and mounted on microslides for a permanent record. The remaining sample was scanned for rare species. Faunal counts for the picked fraction are listed in Tables 1, 2 and 3 . Index species are shown in Plate 1.

TABL. 1. - Relative abundance of K/T boundary planktic foraminifera at Agost.

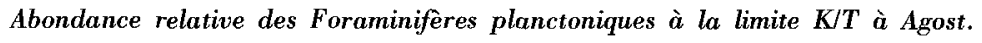




\begin{tabular}{|c|c|c|c|c|c|c|c|c|c|c|c|c|}
\hline \multirow{2}{*}{ SPECIES } & \multicolumn{12}{|c|}{ SAMPLES $>63 \mu$} \\
\hline & $-220-225$ & $-180-185$ & $-140-145$ & $-100-105$ & $.60-65$ & $-30-35$ & $-15-20$ & $-5 \cdot 10$ & 0.2 & $+1+2$ & $+2+3$ & $+3+4$ \\
\hline $\begin{array}{l}\text { Guembelitria cretacea } \\
\text { G. trifolia }\end{array}$ & $\begin{array}{c}0.63 \\
x\end{array}$ & $\begin{array}{l}0.93 \\
0.31\end{array}$ & $\begin{array}{c}0.90 \\
\times\end{array}$ & $\begin{array}{l}0.93 \\
0.62\end{array}$ & $\begin{array}{l}1.83 \\
0.91\end{array}$ & $\begin{array}{l}1.23 \\
0.31\end{array}$ & $\begin{array}{c}0.63 \\
x\end{array}$ & 0.32 & $\begin{array}{c}0.31 \\
\times\end{array}$ & $\begin{array}{l}1.55 \\
0.62\end{array}$ & $\begin{array}{l}2.31 \\
0.66\end{array}$ & $\begin{array}{l}4.23 \\
1.95\end{array}$ \\
\hline $\begin{array}{l}\text { H. planata } \\
\text { H. globulosa } \\
\text { H. pulchra } \\
\text { H. punctulata } \\
\text { H. glabrans } \\
\text { H. labellosa } \\
\text { H. navarroensis } \\
\text { H. carinata } \\
\text { H. semicostata }\end{array}$ & $\begin{array}{c}3.49 \\
38.09 \\
2.22 \\
3.17 \\
0.63 \\
1.27 \\
13.65 \\
1.90 \\
x\end{array}$ & $\begin{array}{c}3.70 \\
37.0 .3 \\
2.78 \\
0.62 \\
0.62 \\
0.31 \\
14.20 \\
0.9 .3 \\
x\end{array}$ & $\begin{array}{c}2.99 \\
+1.50 \\
3.28 \\
0.90 \\
0.90 \\
0.90 \\
14.03 \\
1.49 \\
\times\end{array}$ & $\begin{array}{c}5.86 \\
36.42 \\
2.78 \\
2.16 \\
0.63 \\
0.31 \\
11.42 \\
1.54 \\
x\end{array}$ & $\begin{array}{c}1.35 \\
38.10 \\
4.57 \\
0.91 \\
0.30 \\
2.74 \\
15.24 \\
1.52 \\
x\end{array}$ & $\begin{array}{c}2.77 \\
42.46 \\
2.78 \\
0.92 \\
1.54 \\
0.62 \\
9.54 \\
0.31 \\
\times\end{array}$ & \begin{tabular}{c|}
3.77 \\
39.31 \\
3.14 \\
2.20 \\
1.89 \\
2.20 \\
14.78 \\
0.94
\end{tabular} & $\begin{array}{c}2.24 \\
30.99 \\
2.88 \\
x \\
0.96 \\
2.24 \\
23.64 \\
0.32 \\
x\end{array}$ & $\begin{array}{c}4.70 \\
42.64 \\
2.51 \\
0.94 \\
1.25 \\
1.25 \\
14.73 \\
0.63 \\
x\end{array}$ & $\begin{array}{c}5.57 \\
34.06 \\
0.93 \\
x \\
3.41 \\
5.57\end{array}$ & $\begin{array}{c}3.96 \\
39.60 \\
1.32 \\
\\
0.33 \\
2.97 \\
6.93\end{array}$ & $\begin{array}{r}4.56 \\
43.00 \\
1.63 \\
\\
2.28 \\
4.23\end{array}$ \\
\hline $\begin{array}{l}\text { Pseudotextularia nuttalli } \\
\text { P. elega ns } \\
\text { P. infermedia }\end{array}$ & $\begin{array}{c}1.90 \\
0.32 \\
x\end{array}$ & $\begin{array}{l}1.23 \\
1.23 \\
0.31\end{array}$ & $\begin{array}{l}1.79 \\
1.19 \\
0.30\end{array}$ & $\begin{array}{c}2.47 \\
0.93 \\
x\end{array}$ & $\begin{array}{l}0.61 \\
0.91 \\
0.30\end{array}$ & $\begin{array}{c}1.23 \\
0.31 \\
x\end{array}$ & $\begin{array}{l}1.26 \\
0.31 \\
0.31\end{array}$ & $\begin{array}{c}0.96 \\
0.32 \\
x\end{array}$ & $\begin{array}{c}2.19 \\
0.94 \\
x\end{array}$ & & & \\
\hline $\begin{array}{l}\text { Gublerina acuta } \\
\text { G. cuvillieri }\end{array}$ & $\begin{array}{l}\mathbf{x} \\
\mathbf{x}\end{array}$ & $\begin{array}{c}0.31 \\
x\end{array}$ & $\begin{array}{c}0.30 \\
x\end{array}$ & $\begin{array}{c}x \\
0.31\end{array}$ & $\mathbf{x}$ & $\begin{array}{l}\mathbf{x} \\
\mathbf{x}\end{array}$ & $\mathbf{x}$ & $\mathbf{x}$ & $\mathbf{x}$ & & & \\
\hline $\begin{array}{l}\text { Pseudoguembelina kempensis } \\
\text { P. palpebra } \\
\text { P. costulata } \\
\text { P. costellifera } \\
\text { P. excolata } \\
\text { P. hariaensis }\end{array}$ & $\begin{array}{l}2.22 \\
0.63 \\
2.86 \\
\mathrm{x} \\
\mathrm{x} \\
\mathrm{x}\end{array}$ & $\begin{array}{l}1.23 \\
1.23 \\
2.47 \\
0.62 \\
0.31\end{array}$ & $\begin{array}{l}0.90 \\
0.60 \\
3.28 \\
0.30 \\
\times \\
\mathrm{x}\end{array}$ & $\begin{array}{l}+.01 \\
1.2 .3 \\
2.47 \\
\times \\
\mathrm{x} \\
0.62\end{array}$ & $\begin{array}{l}1.52 \\
0.61 \\
2.74 \\
\mathrm{x} \\
\mathrm{x}\end{array}$ & $\begin{array}{l}2.77 \\
0.62 \\
3.69 \\
\times \\
x\end{array}$ & $\begin{array}{c}1.89 \\
x \\
3.14 \\
0.31 \\
x \\
x\end{array}$ & $\begin{array}{c}1.92 \\
0.64 \\
8.31 \\
\mathrm{x} \\
\mathrm{x} \\
0.32\end{array}$ & $\begin{array}{l}2.82 \\
0.31 \\
5.33 \\
x \\
x \\
x\end{array}$ & $\begin{array}{l}3.41 \\
4.64\end{array}$ & $\begin{array}{l}7.26 \\
4.95\end{array}$ & $\begin{array}{l}6.19 \\
1.63\end{array}$ \\
\hline $\begin{array}{l}\text { Planoglobulina acervulinoides } \\
\text { P. carseyae } \\
\text { P. multicamerata } \\
\text { P. manuelensis }\end{array}$ & $\begin{array}{l}\mathbf{x} \\
\mathbf{x} \\
\mathbf{x} \\
\mathbf{x}\end{array}$ & $\begin{array}{c}x \\
0.62 \\
0.31\end{array}$ & $\begin{array}{c}\mathrm{x} \\
0.30 \\
0.30 \\
\mathrm{x}\end{array}$ & $\frac{x}{0.31}$ & $\begin{array}{l}\mathbf{x} \\
\mathbf{x} \\
\mathbf{x}\end{array}$ & $\begin{array}{l}\mathbf{x} \\
\mathbf{x} \\
\mathbf{x}\end{array}$ & $\begin{array}{c}x \\
0.31 \\
x\end{array}$ & $\begin{array}{l}\mathbf{x} \\
\mathbf{x} \\
\mathbf{x} \\
\mathbf{x}\end{array}$ & $\begin{array}{l}\mathbf{x} \\
\mathbf{x} \\
\mathbf{x} \\
\mathbf{x}\end{array}$ & & & \\
\hline $\begin{array}{l}\text { Racemiguembelina fructicosa } \\
\text { R. powelli }\end{array}$ & $\mathbf{x}$ & $\begin{array}{c}0.31 \\
x\end{array}$ & $\begin{array}{l}0.30 \\
0.60\end{array}$ & $\begin{array}{c}x \\
0.31\end{array}$ & $\mathbf{x}$ & $\begin{array}{c}0.31 \\
x\end{array}$ & $\mathbf{x}$ & $\underset{0.32}{x}$ & $\stackrel{x}{0.31}$ & & & \\
\hline $\begin{array}{l}\text { Globigerinelloides y aucoensis } \\
\text { G. rosebudensis } \\
\text { G. prairiehillensis } \\
\text { G. volutus } \\
\text { G. subearinatus }\end{array}$ & $\begin{array}{l}0.95 \\
0.32 \\
3.49 \\
0.63 \\
0.95\end{array}$ & $\begin{array}{l}3.40 \\
0.62 \\
3.09 \\
0.93 \\
0.93\end{array}$ & $\begin{array}{l}1.79 \\
0.30 \\
2.39 \\
0.30 \\
0.30\end{array}$ & $\begin{array}{l}0.93 \\
0.31 \\
5.56 \\
0.62 \\
0.31\end{array}$ & $\begin{array}{l}1.52 \\
x \\
3.05 \\
x \\
x\end{array}$ & $\begin{array}{l}2.15 \\
\mathrm{x} \\
3.69 \\
0.61 \\
\mathrm{x}\end{array}$ & $\begin{array}{c}1.57 \\
x \\
3.77 \\
0.63 \\
x\end{array}$ & $\begin{array}{c}4.47 \\
x \\
3.51 \\
1.28 \\
0.64\end{array}$ & $\begin{array}{c}2.82 \\
0.31 \\
3.13 \\
1.89 \\
\mathrm{x}\end{array}$ & $\begin{array}{l}3.72 \\
\\
7.12 \\
3.10 \\
0.62\end{array}$ & $\begin{array}{l}3.30 \\
\\
6.93 \\
2.31 \\
0.33 \\
.33\end{array}$ & $\begin{array}{l}2.61 \\
4.89 \\
3.26 \\
x\end{array}$ \\
\hline $\begin{array}{l}\text { Hedbergella monmouthensis } \\
\text { H. holmdelensis }\end{array}$ & $\begin{array}{l}3.81 \\
1.59\end{array}$ & $\begin{array}{l}7.72 \\
2.78\end{array}$ & $\begin{array}{l}5.67 \\
2.39\end{array}$ & $\begin{array}{l}3.40 \\
1.85\end{array}$ & $\begin{array}{l}7.32 \\
6.40\end{array}$ & $\begin{array}{l}9.23 \\
4.31\end{array}$ & $\begin{array}{l}8.81 \\
2.20\end{array}$ & $\begin{array}{l}5.75 \\
2.24\end{array}$ & $\begin{array}{l}2.51 \\
2.19\end{array}$ & $\begin{array}{l}6.50 \\
10.53\end{array}$ & $\begin{array}{l}3.63 \\
9.24\end{array}$ & $\begin{array}{l}7.82 \\
9.77\end{array}$ \\
\hline $\begin{array}{l}\text { Globotruncanella caravacaensis } \\
\text { G. havanensis } \\
\text { G. petaloidea } \\
\text { G. pschadae } \\
\text { G. minuta }\end{array}$ & $\begin{array}{c}0.32 \\
x \\
0.65 \\
0.32 \\
2.22\end{array}$ & $\begin{array}{c}x \\
0.31 \\
0.31\end{array}$ & $\begin{array}{c}x \\
x \\
0.30 \\
x \\
1.49\end{array}$ & $\begin{array}{c}0.9 .3 \\
x \\
x \\
x \\
2.16\end{array}$ & $\begin{array}{c}0.30 \\
x \\
x \\
x \\
0.61\end{array}$ & $\begin{array}{c}0.31 \\
x \\
0.62 \\
x \\
1.23\end{array}$ & $\begin{array}{c}0.63 \\
x \\
x \\
x \\
0.94\end{array}$ & $\begin{array}{c}0.32 \\
\mathbf{x} \\
\mathbf{x} \\
\mathbf{x} \\
1.92\end{array}$ & $\begin{array}{c}0.31 \\
\mathbf{x} \\
\mathbf{x} \\
\mathbf{x} \\
0.94\end{array}$ & 4.02 & 2.31 & 1.30 \\
\hline $\begin{array}{l}\text { Archaeoglobigerina cretacea } \\
\text { A. blowi }\end{array}$ & $\begin{array}{l}0.32 \\
0.32\end{array}$ & $\underset{0.31}{x}$ & $\mathbf{x}$ & 0.62 & $\begin{array}{c}0.30 \\
x\end{array}$ & $\begin{array}{l}0.31 \\
0.31\end{array}$ & $\mathbf{x}$ & 0.32 & 0.31 & & & \\
\hline Schackoina multispinata & 0.32 & 0.62 & 1.19 & $\mathrm{x}$ & 0.61 & 0.31 & 0.31 & 1.28 & 0.31 & & & \\
\hline Plummerita hantkeninoides & 0.32 & 0.31 & $\mathbf{x}$ & $\mathbf{x}$ & 0.30 & 0.31 & 0.31 & & $\mathbf{x}$ & & & \\
\hline $\begin{array}{l}\text { Rugoglobigerina reicheli } \\
\text { R. rugosa } \\
\text { R. hexacamerata } \\
\text { R. rotundata } \\
\text { R. milamensis } \\
\text { R. pennyi } \\
\text { R. macrocephala } \\
\text { R. scotti }\end{array}$ & $\begin{array}{c}0.65 \\
1.27 \\
0.32 \\
\mathbf{x} \\
\mathrm{x} \\
\mathrm{x} \\
\mathrm{x} \\
1.27\end{array}$ & $\begin{array}{c}0.93 \\
0.93 \\
0.62 \\
\mathrm{x} \\
\mathrm{x} \\
\mathrm{x} \\
\mathrm{x} \\
0.31\end{array}$ & $\begin{array}{l}0.30 \\
0.40 \\
1.49 \\
x \\
x \\
x \\
x \\
0.60\end{array}$ & $\begin{array}{c}1.23 \\
2.47 \\
0.31 \\
x \\
\times \\
0.31 \\
0.31 \\
1.23\end{array}$ & $\begin{array}{c}0.61 \\
0.61 \\
\mathrm{x} \\
\mathrm{x} \\
\mathrm{x} \\
\mathrm{x} \\
0.30\end{array}$ & $\begin{array}{c}0.31 \\
0.31 \\
x \\
x \\
\\
x \\
0.92\end{array}$ & $\begin{array}{c}0.63 \\
0.94 \\
0.63 \\
\times \\
\times \\
0.31 \\
0.31\end{array}$ & $\begin{array}{l}0.64 \\
\mathrm{x} \\
\mathrm{x} \\
\mathrm{x} \\
\mathrm{x} \\
\mathrm{x}\end{array}$ & $\begin{array}{c}0.31 \\
0.94 \\
0.31 \\
\times \\
\\
\times \\
0.63\end{array}$ & & & \\
\hline $\begin{array}{l}\text { Globotruncana arca } \\
\text { G. uegyptiaca } \\
\text { G. rosetta } \\
\text { G. marici }\end{array}$ & $\begin{array}{l}1.59 \\
0.95 \\
0.32 \\
1.59\end{array}$ & $\begin{array}{c}0.62 \\
1.23 \\
x \\
0.62\end{array}$ & $\begin{array}{c}0.90 \\
0.30 \\
x \\
0.90\end{array}$ & $\begin{array}{l}0.31 \\
0.31 \\
0.31 \\
0.31\end{array}$ & $\begin{array}{c}0.30 \\
0.30 \\
x \\
0.30\end{array}$ & $\begin{array}{c}x \\
0.92 \\
0.31 \\
0.31\end{array}$ & $\begin{array}{c}\mathrm{x} \\
0.31 \\
0.63\end{array}$ & $\begin{array}{c}0.32 \\
x \\
x \\
0.64\end{array}$ & $\begin{array}{c}0.63 \\
0.63 \\
0.31 \\
x\end{array}$ & & & \\
\hline $\begin{array}{l}\text { Globotruncanita stuarti } \\
\text { Gita. stuartiform is } \\
\text { Gita. insignis } \\
\text { Gita. fareedi } \\
\text { Gita. conica } \\
\text { Gita. elevata } \\
\text { Gita. falsocalcarata } \\
\text { Gita. dupeublei }\end{array}$ & $\begin{array}{c}0.32 \\
0.32 \\
x \\
1.27 \\
x \\
x \\
x \\
x\end{array}$ & $\begin{array}{c}x \\
0.31 \\
0.62 \\
x \\
0.31 \\
x\end{array}$ & $\begin{array}{c}0.30 \\
0.60 \\
x \\
0.60 \\
x \\
x \\
x \\
x\end{array}$ & $\begin{array}{c}0.31 \\
x \\
x \\
0.31 \\
0.31 \\
x \\
x\end{array}$ & $\begin{array}{c}0.30 \\
x \\
x \\
0.30 \\
x \\
x \\
x \\
x\end{array}$ & $\begin{array}{c}\mathrm{x} \\
0.62 \\
\mathrm{x} \\
1.23 \\
\mathrm{x} \\
\mathrm{x} \\
0.31 \\
\mathrm{x}\end{array}$ & $\begin{array}{c}\mathbf{x} \\
\mathbf{x} \\
\mathbf{x} \\
\mathbf{0 . 3 1} \\
\mathbf{x} \\
\mathbf{x} \\
\mathbf{x} \\
\mathbf{x}\end{array}$ & $\begin{array}{c}\mathrm{x} \\
0.32 \\
\mathrm{x} \\
\mathrm{x} \\
\mathrm{x} \\
\mathrm{x} \\
\mathrm{x}\end{array}$ & $\begin{array}{c}\mathbf{x} \\
\mathbf{x} \\
\mathbf{x} \\
0.31 \\
\mathbf{x} \\
\mathbf{x} \\
0.31 \\
\mathbf{x}\end{array}$ & & & \\
\hline $\begin{array}{l}\text { Contusotruncana contusa } \\
\text { C. palelliformis } \\
\text { C. walfischensis }\end{array}$ & $\begin{array}{l}\mathbf{x} \\
\mathbf{x} \\
\mathbf{x}\end{array}$ & $\begin{array}{l}\mathrm{x} \\
\mathrm{x} \\
\mathrm{x}\end{array}$ & $\begin{array}{c}\mathrm{x} \\
\mathbf{0 . 3 0}\end{array}$ & $\mathbf{x}$ & $\begin{array}{c}0.30 \\
x\end{array}$ & $\begin{array}{l}x \\
x \\
x\end{array}$ & $\begin{array}{l}x \\
x \\
x\end{array}$ & $\mathrm{x}$ & $\begin{array}{l}\mathbf{x} \\
\mathbf{x}\end{array}$ & & & \\
\hline $\begin{array}{l}\text { Abhatomphalus mayaroensis } \\
\text { A. intermedius }\end{array}$ & $\mathbf{x}$ & $\mathrm{x}$ & $\mathrm{x}$ & $\mathbf{x}$ & $\mathrm{x}$ & $\mathrm{x}$ & $\begin{array}{l}\mathbf{x} \\
\mathbf{x}\end{array}$ & $\mathrm{x}$ & $\begin{array}{l}x \\
x\end{array}$ & & & \\
\hline TOTAL NUMBER COUNTED & 315 & 324 & 335 & 324 & 328 & 325 & 318 & 313 & 319 & 323 & 303 & 307 \\
\hline
\end{tabular}


TABL. 2. - Relative abundance of lowermost Danian planktic forminifera at Agost. Abondance relative des Foraminiferes planctoniques au Danien inférieur à Agost

\begin{tabular}{|c|c|c|c|c|c|c|c|c|c|c|c|c|c|c|c|c|}
\hline \multirow{2}{*}{ SPECIES } & \multicolumn{16}{|c|}{ SAMPLES > $63 \mu$} \\
\hline & $+4+6$ & $+6+8$ & $+8+10$ & $+10+12$ & $+12+14$ & $+14+16$ & $+16+18$ & $+18+20$ & $+20+22$ & $+22+24$ & $+24+26$ & $+26+28$ & $+28+30$ & $+35+40$ & $+45+47$ & $+65+70$ \\
\hline $\begin{array}{l}\text { Parvularugoglob. longiapertura } \\
\text { P. sabina } \\
\text { P. umbrica } \\
\text { P. eugubinat } \\
\text { P. theodosica } \\
\text { P. hemisphaerica }\end{array}$ & 7.97 & $\begin{array}{r}36.55 \\
0.84 \\
4.62\end{array}$ & $\begin{array}{r}59.85 \\
1.46 \\
9.85\end{array}$ & $\begin{array}{c}73.69 \\
1.53 \\
4.89\end{array}$ & $\begin{array}{c}68.26 \\
3.69 \\
5.90 \\
0.73\end{array}$ & $\begin{array}{l}6.3 .60 \\
8.25 \\
2.14 \\
3.97 \\
0.9 ! \\
0.91\end{array}$ & $\begin{array}{l}49.50 \\
15.19 \\
1.58 \\
6.33 \\
4.74 \\
0.63\end{array}$ & $\begin{array}{c}41.97 \\
18.48 \\
0.36 \\
10.95 \\
4.37 \\
0.36\end{array}$ & $\begin{array}{r}22.25 \\
10.06 \\
0.61 \\
17.07 \\
4.26 \\
1.22\end{array}$ & $\begin{array}{c}12.65 \\
6.02 \\
\\
10.54 \\
5.12 \\
0.90\end{array}$ & \begin{tabular}{c|}
13.79 \\
7.93 \\
\\
10.69 \\
5.51 \\
1.03
\end{tabular} & \begin{tabular}{c|}
10.93 \\
8.98 \\
10.15 \\
14.06 \\
0.39
\end{tabular} & $\begin{array}{c}10.30 \\
9.54 \\
9.92 \\
7.63 \\
0.76\end{array}$ & $\begin{array}{c}6.38 \\
5.10 \\
16.59 \\
12.34 \\
1.27\end{array}$ & $\begin{array}{c}20.68 \\
12,41 \\
3.44\end{array}$ & $\begin{array}{l}10.71 \\
2.67 \\
0.89\end{array}$ \\
\hline $\begin{array}{l}\text { Globoconusa fodina } \\
\text { G. exlensa } \\
\text { G. conusa } \\
\text { G. minutula }\end{array}$ & $\begin{array}{l}3.32 \\
10.63\end{array}$ & $\begin{array}{l}2.10 \\
3.78 \\
+.62 \\
1.26\end{array}$ & $\begin{array}{l}2.55 \\
1.32 \\
8.39 \\
x\end{array}$ & $\begin{array}{c}2.14 \\
1.53 \\
7.34 \\
x\end{array}$ & $\begin{array}{l}5.16 \\
1.47 \\
1.84 \\
2.95\end{array}$ & $\begin{array}{l}3.66 \\
2.75 \\
2.44 \\
1.22\end{array}$ & $\begin{array}{l}6.33 \\
7.59 \\
2.84 \\
0.95\end{array}$ & $\begin{array}{c}5.84 \\
1.46 \\
\times \\
0.36\end{array}$ & $\begin{array}{l}5.18 \\
0.91 \\
2.13 \\
0.30\end{array}$ & $\begin{array}{l}7.22 \\
1.20 \\
2.71 \\
0.90\end{array}$ & $\begin{array}{l}7.24 \\
0.69 \\
1.72 \\
0.34\end{array}$ & $\begin{array}{c}8.20 \\
0.78 \\
1.56 \\
x\end{array}$ & $\begin{array}{l}7.24 \\
4.58 \\
6.48 \\
1.14\end{array}$ & $\begin{array}{l}4.25 \\
0.42 \\
3.83 \\
0.85\end{array}$ & $\begin{array}{l}2.06 \\
1.38 \\
1.38\end{array}$ & 1.73 \\
\hline $\begin{array}{l}\text { Eoglobigerina simplicissima } \\
\text { E. eobulloides } \\
\text { E. pentagona } \\
\text { E. fringa } \\
\text { E. trivialis } \\
\text { E. praedita } \\
\text { E. edita }\end{array}$ & & & & & & & & & & & & 0.39 & $\begin{array}{c}x \\
0.76\end{array}$ & $\begin{array}{r}0.42 \\
1.70 \\
\\
2.97\end{array}$ & $\begin{array}{l}0.69 \\
1.38\end{array}$ & $\begin{array}{l}0.89 \\
1.78 \\
\\
3.57 \\
1.78 \\
0.89\end{array}$ \\
\hline Subbotina triloculinoides & & & & & & & & & & & & & & & $0.69 ?$ & 2.67 \\
\hline $\begin{array}{l}\text { Globanomalina danica } \\
\text { G. archeocompresa } \\
\text { G. planocompresa }\end{array}$ & & & & & & & & & & 3.01 & 1.72 & 1.95 & 2.67 & 1.27 & $x$ & 0.89 \\
\hline $\begin{array}{l}\text { Parasubbotina moskvini } \\
\text { P. variantit } \\
\text { P. pseudobulloides } \\
\text { P. inconstans }\end{array}$ & & & & & & & & & & & & & & & 2.06 & 0.89 \\
\hline $\begin{array}{l}\text { Pracmurica tauricat } \\
\text { P. psendoinconstans }\end{array}$ & & & & & & & & & & & & & & & $\begin{array}{c}12.41 \\
1.38\end{array}$ & $\begin{array}{l}6.25 \\
1.78\end{array}$ \\
\hline $\begin{array}{l}\text { Chiloguembelina morsei } \\
\text { Ch. midwayensis } \\
\text { Ch. taurica }\end{array}$ & & & & & & 0.60 & $\begin{array}{l}\mathbf{x} \\
\mathbf{x}\end{array}$ & $\begin{array}{l}5.11 \\
3.28\end{array}$ & $\begin{array}{l}18.29 \\
2.13 \\
7.92\end{array}$ & $\begin{array}{l}28.61 \\
3.01 \\
9.94\end{array}$ & $\begin{array}{c}31.72 \\
2.06 \\
13.44\end{array}$ & $\begin{array}{c}26.17 \\
2.73 \\
8.20\end{array}$ & $\begin{array}{c}19.84 \\
0.76 \\
10.30\end{array}$ & $\begin{array}{c}17.87 \\
0.85 \\
15.74\end{array}$ & $\begin{array}{l}18.62 \\
4.13\end{array}$ & $\begin{array}{l}38.39 \\
2.67 \\
8.03\end{array}$ \\
\hline $\begin{array}{l}\text { Woodringina claytonensis } \\
\text { W. hornerstownensis }\end{array}$ & & 0.42 & $\begin{array}{l}1.46 \\
1.46\end{array}$ & $\mathbf{x}$ & $\begin{array}{c}x \\
0.36\end{array}$ & $\begin{array}{c}0.30 \\
x\end{array}$ & $\begin{array}{l}\mathbf{x} \\
\mathbf{x}\end{array}$ & $\begin{array}{l}0.36 \\
1.09\end{array}$ & $\begin{array}{l}0.91 \\
0.91\end{array}$ & $\begin{array}{l}2.10 \\
2.41\end{array}$ & $\begin{array}{c}1.72 \\
x\end{array}$ & $\begin{array}{l}1.56 \\
1.56\end{array}$ & $\begin{array}{l}3.05 \\
1.91\end{array}$ & $\begin{array}{l}0.85 \\
0.42\end{array}$ & $\begin{array}{l}0.62 \\
2.06\end{array}$ & $\begin{array}{l}3.57 \\
1.78\end{array}$ \\
\hline $\begin{array}{l}\text { Guembelitria danica } \\
\text { G. Irregularis } \\
\text { G. alabamensis } \\
\text { G. cretacea } \\
\text { G. trifolia }\end{array}$ & $\begin{array}{r}2.33 \\
0.66 \\
0.66 \\
16.28 \\
4.65\end{array}$ & $\begin{array}{c}5.46 \\
4.80 \\
x \\
6.30 \\
0.84\end{array}$ & $\begin{array}{c}1.82 \\
\times \\
\times \\
3.28 \\
2.19\end{array}$ & $\begin{array}{c}x \\
0.91 \\
\\
3.97 \\
0.31\end{array}$ & $\begin{array}{c}0.73 \\
0.73 \\
x \\
2.95 \\
0.36\end{array}$ & $\begin{array}{l}0.61 \\
0.61 \\
3.67 \\
0.30\end{array}$ & $\begin{array}{c}0.63 \\
x \\
x \\
1.58 \\
x\end{array}$ & $\begin{array}{l}0.36 \\
1.46 \\
1.09\end{array}$ & $\begin{array}{c}x \\
x \\
0.91 \\
1.82 \\
x\end{array}$ & $\begin{array}{c}x \\
0.90 \\
0.30\end{array}$ & $\begin{array}{c}x \\
x \\
x \\
0.69 \\
0.34\end{array}$ & $\begin{array}{c}0.78 \\
x \\
0.39 \\
0.39\end{array}$ & $\begin{array}{c}x \\
0.76 \\
0.38 \\
0.38 \\
1.52\end{array}$ & $\begin{array}{c}x \\
\mathbf{x} \\
\mathbf{x} \\
0.85 \\
\mathrm{x}\end{array}$ & 0.69 & 0.89 \\
\hline $\begin{array}{l}\text { Heterohelis planata } \\
\text { H. globulosa } \\
\text { H. pulchra } \\
\text { II. naviaroensis }\end{array}$ & \begin{tabular}{r|}
1.99 \\
11.96 \\
1.66 \\
2.99
\end{tabular} & $\begin{array}{l}0.42 \\
4.62 \\
1.26 \\
2.10\end{array}$ & $\begin{array}{c}0.37 \\
1.46 \\
x \\
0.73\end{array}$ & $\begin{array}{l}x \\
0.92 \\
0.31 \\
0.31\end{array}$ & $\begin{array}{c}x \\
0.37 \\
x\end{array}$ & $\begin{array}{c}x \\
x \\
0.61\end{array}$ & $\begin{array}{l}0.95 \\
0.95 \\
0.32 \\
0.32\end{array}$ & $\mathbf{x}$ & $\begin{array}{l}x \\
x\end{array}$ & $\begin{array}{l}0.60 \\
0.30 \\
0.90\end{array}$ & $\begin{array}{l}\mathbf{x} \\
\mathbf{x} \\
\mathbf{x} \\
\mathbf{x}\end{array}$ & $\begin{array}{c}x \\
0.39 \\
0.39\end{array}$ & $\begin{array}{l}\mathbf{x} \\
\mathbf{x}\end{array}$ & $\begin{array}{c}0.43 \\
x \\
0.43\end{array}$ & & $\begin{array}{l}0.89 \\
1.78\end{array}$ \\
\hline $\begin{array}{l}\text { Pscudogienbelina kempensis } \\
\text { P. costulata }\end{array}$ & $\begin{array}{l}1.99 \\
1.00\end{array}$ & $x$ & $\mathbf{x}$ & 0.31 & $x$ & $\mathbf{x}$ & 0.32 & & $\mathbf{x}$ & & & & & & & \\
\hline $\begin{array}{l}\text { Globigerinelloides yaucoensis } \\
\text { G. prairiehilkensis } \\
\text { G. volulus } \\
\text { G. subcarinatus }\end{array}$ & $\begin{array}{l}4.32 \\
3.99 \\
2.33 \\
0.66\end{array}$ & $\begin{array}{l}2.94 \\
3.78 \\
2.10 \\
x\end{array}$ & $\begin{array}{l}0.73 \\
0.37 \\
0.37\end{array}$ & $\begin{array}{c}0.31 \\
0.31 \\
x\end{array}$ & $\begin{array}{l}1.11 \\
0.37 \\
0.37\end{array}$ & $\begin{array}{c}x \\
x \\
0.61\end{array}$ & $\begin{array}{l}x^{*} \\
x\end{array}$ & $\begin{array}{l}\mathbf{x} \\
\mathbf{x} \\
\mathbf{x}\end{array}$ & & $\begin{array}{c}0.60 \\
x\end{array}$ & $\mathbf{x}$ & $\begin{array}{l}\mathbf{x} \\
\mathbf{x}\end{array}$ & & $\mathbf{x}$ & & \\
\hline $\begin{array}{l}\text { Hedbergella monmouthensis } \\
\text { II. holmdelensis }\end{array}$ & $\begin{array}{l}5.98 \\
11.63\end{array}$ & $\begin{array}{l}5.04 \\
3.36\end{array}$ & $\begin{array}{l}1.10 \\
0.37\end{array}$ & $\begin{array}{c}0.31 \\
x\end{array}$ & $\begin{array}{l}1.11 \\
0.74\end{array}$ & 0.31 & $\begin{array}{c}\mathrm{x} \\
0.95\end{array}$ & 0.37 & $\begin{array}{l}\mathbf{x} \\
\mathbf{x}\end{array}$ & $x$ & $\mathrm{x}$ & $\mathbf{x}$ & $\mathbf{x}$ & $x$ & 0.69 & 0.89 \\
\hline $\begin{array}{l}\text { Globotruncanella caravaciensis } \\
\text { G. minuta }\end{array}$ & $\begin{array}{l}1.66 \\
1.66\end{array}$ & $\begin{array}{l}0.42 \\
0.42\end{array}$ & $\underset{0.37}{x}$ & 0.31 & $\begin{array}{l}0.37 \\
0.37\end{array}$ & $\begin{array}{l}\mathrm{x} \\
\mathrm{x}\end{array}$ & 0.32 & & & & & & & & & \\
\hline TOTAL NUMBER COLNTED & 301 & 238 & 274 & 327 & 271 & 327 & 315 & 274 & 328 & 332 & 290 & 256 & 262 & 235 & 144 & 112 \\
\hline
\end{tabular}


TaBL. 3. - Relative abundance of lower Danian planktic foraminifera at Agost.

Abondance relative des Foraminifêres planctoniques au Danien infërieur à Agost.

\begin{tabular}{|c|c|c|c|c|c|c|c|c|c|c|c|c|c|c|c|c|c|c|c|c|}
\hline \multirow{2}{*}{ SPECIES } & \multicolumn{20}{|c|}{ SAMPLES $>63 \mu$} \\
\hline & $\begin{array}{l}+82 \\
+86 \\
\end{array}$ & $\begin{array}{l}+98 \\
+102 \\
\end{array}$ & $\begin{array}{r}+114 \\
+118 \\
\end{array}$ & $\begin{array}{r}+130 \\
+134 \\
\end{array}$ & $\begin{array}{l}+146 \\
+148 \\
\end{array}$ & $\begin{array}{l}+160 \\
+164\end{array}$ & $\begin{array}{l}+176 \\
+180 \\
\end{array}$ & $\begin{array}{l}+192 \\
+196\end{array}$ & $\begin{array}{l}+208 \\
+212 \\
\end{array}$ & $\begin{array}{r}+224 \\
+226 \\
\end{array}$ & $\begin{array}{l}+244 \\
+248 \\
\end{array}$ & $\begin{array}{l}+260 \\
+264 \\
+26\end{array}$ & $\begin{array}{l}+284 \\
+289 \\
\end{array}$ & $\begin{array}{l}+309 \\
+314 \\
\end{array}$ & $\begin{array}{l}+334 \\
+339 \\
\end{array}$ & $\begin{array}{l}+364 \\
+369 \\
\end{array}$ & $\begin{array}{r}+404 \\
+409 \\
\end{array}$ & $\begin{array}{r}+434 \\
+439 \\
\end{array}$ & $\begin{array}{l}+464 \\
+469 \\
\end{array}$ & $\begin{array}{l}+489 \\
+494 \\
\end{array}$ \\
\hline Globastica daubjergensis & & & & & & & & & $\mathrm{x}$ & 0.33 & 0.72 & $\mathrm{x}$ & $\mathrm{x}$ & 0.61 & 2.86 & $\mathrm{~s}$ & 0.70 & $\mathbf{x}$ & 0.72 & 4.71 \\
\hline $\begin{array}{l}\text { Eoglobigerina simplicissima } \\
\text { En eobulloides } \\
\text { E. pentagona } \\
\text { E. fringa } \\
\text { E. Irivialis } \\
\text { E. preadita } \\
\text { E. edita } \\
\text { E. polycamera } \\
\text { E. tetragona }\end{array}$ & $\begin{array}{c}0.99 \\
0.66 \\
\\
x \\
x \\
0.66\end{array}$ & $\begin{array}{c}x \\
0.35 \\
x \\
x \\
0.35 \\
x\end{array}$ & $\begin{array}{c}x \\
x \\
0.62 \\
0.31 \\
x\end{array}$ & $\begin{array}{c}0.82 \\
0.27 \\
\\
0.27 \\
x \\
1.37 \\
x\end{array}$ & $\begin{array}{c}0.93 \\
0.62 \\
\\
1.55 \\
x \\
x \\
1.55\end{array}$ & $\begin{array}{l}0.30 \\
0.92 \\
1.23 \\
0.61 \\
1.23\end{array}$ & $\begin{array}{c}\mathrm{x} \\
\mathrm{x} \\
0.74 \\
2.60 \\
1.11 \\
1.85 \\
1.85\end{array}$ & $\begin{array}{c}0.29 \\
0.29 \\
x \\
1 .+8 \\
x \\
0.89 \\
1.18\end{array}$ & $\begin{array}{l}2.16 \\
1.23 \\
0.30 \\
2.77 \\
0.30 \\
0.92 \\
3.08\end{array}$ & $\begin{array}{c}0.33 \\
\mathbf{x} \\
0.33 \\
0.33 \\
1.69 \\
2.02 \\
1.01 \\
\mathbf{x}\end{array}$ & $\begin{array}{c}0.36 \\
1.08 \\
x \\
0.36 \\
0.36 \\
0.36 \\
1.08 \\
0.36 \\
\mathbf{x}\end{array}$ & $\begin{array}{c}x \\
0.91 \\
x \\
1.94 \\
0.97 \\
1.94 \\
2.92 \\
0.65 \\
x\end{array}$ & $\begin{array}{c}x \\
2.84 \\
x \\
1.26 \\
0.63 \\
1.86 \\
.1 .19 \\
0.94 \\
x\end{array}$ & $\begin{array}{c}x \\
1.53 \\
0.61 \\
2.44 \\
2.14 \\
4.58 \\
3.36 \\
x \\
0.30\end{array}$ & $\begin{array}{l}1.29 \\
0.64 \\
0.32 \\
0.97 \\
1.94 \\
3.88 \\
4.53 \\
0.64 \\
0.64\end{array}$ & $\begin{array}{l}1.09 \\
0.36 \\
0.36 \\
0.36 \\
1.45 \\
1.81 \\
3.63 \\
\mathrm{x} \\
\mathrm{x}\end{array}$ & $\begin{array}{c}0.35 \\
x \\
0.35 \\
1.40 \\
2.80 \\
4.91 \\
0.70 \\
x\end{array}$ & $\begin{array}{l}0.33 \\
0.33 \\
0.98 \\
1.64 \\
3.29 \\
2.30 \\
0.98 \\
0.33\end{array}$ & $\begin{array}{c}0.36 \\
0.36 \\
1.81 \\
3.98 \\
3.62 \\
4.71 \\
x \\
0.72\end{array}$ & $\begin{array}{l}0.31 \\
x \\
3.46 \\
2.51 \\
1.25 \\
1.57 \\
0.62 \\
0.31\end{array}$ \\
\hline Subbotina triloculinoides & $0.66 ?$ & 0.70 ? & $0.31 ?$ & t.10: & $1.55 ?$ & 1.23 & 1.11 & $\mathrm{x}$ & 1.85 & 0.67 & 0.36 & $\mathrm{~s}$ & 0.94 & 0.30 & 0.32 & $\mathrm{~s}$ & $\mathrm{~s}$ & $\mathrm{x}$ & 0.36 & 5.03 \\
\hline $\begin{array}{l}\text { Globanomalina danica } \\
\text { G. archeocompresa } \\
\text { G. planocompresa } \\
\text { G. eompressa } \\
\text { G. caucasica }\end{array}$ & $\mathrm{x}$ & $\begin{array}{c}1 .+0 \\
x\end{array}$ & $\begin{array}{c}0.31 \\
x\end{array}$ & $\begin{array}{c}1.10 \\
\mathrm{x}\end{array}$ & $\begin{array}{l}4.34 \\
0.31 \\
x\end{array}$ & $\begin{array}{l}2.15 \\
0.30 \\
\mathrm{x}\end{array}$ & $\begin{array}{l}6.65 \\
0.74 \\
2.23 \\
1.11\end{array}$ & $\begin{array}{c}x \\
0.59 \\
0.29 \\
0.29\end{array}$ & $\begin{array}{l}1.23 \\
0.61 \\
0.61 \\
\\
5.24\end{array}$ & $\begin{array}{l}1.35 \\
6.33 \\
1.35 \\
2.02\end{array}$ & $\begin{array}{l}1.80 \\
2.88 \\
3.24 \\
0.36\end{array}$ & $\begin{array}{l}2.27 \\
15.90 \\
7.79 \\
0.97\end{array}$ & $\begin{array}{l}2.84 \\
12.61 \\
+.10 \\
1.57\end{array}$ & $\begin{array}{l}1.53 \\
7.34 \\
+.88 \\
\mathrm{x}\end{array}$ & $\begin{array}{c}0.64 \\
9.71 \\
5.17 \\
2.91 \% \\
x\end{array}$ & $\begin{array}{c}0.36 \\
4.36 \\
0.36 \\
0.72 ? \\
x\end{array}$ & $\begin{array}{c}2.45 \\
17.54 \\
3.15 \\
3.15 ? \\
\mathrm{x}\end{array}$ & $\begin{array}{l}x \\
5.92 \\
1.97 \\
2.63 \\
0.33\end{array}$ & $\begin{array}{c}0.36 \\
8.69 \\
2.17 \\
1.81 \\
x\end{array}$ & $\begin{array}{c}x \\
9.12 \\
3.14 \\
3.77 \\
2.51\end{array}$ \\
\hline $\begin{array}{l}\text { Parasubbotina moskvini } \\
\text { P. varianta } \\
\text { P. pseudabulloides } \\
\text { P. inconstans }\end{array}$ & $\begin{array}{c}0.99 \\
x \\
x\end{array}$ & $\begin{array}{c}0.70 \\
0.35 \\
x\end{array}$ & $\begin{array}{c}x \\
x \\
0.31\end{array}$ & $\begin{array}{l}0.27 \\
0.27 \\
0.55\end{array}$ & $\begin{array}{c}1.55 \\
x \\
0.62 \\
x\end{array}$ & $\begin{array}{c}0.30 \\
x \\
0.61\end{array}$ & $\begin{array}{c}1.11 \\
0.37 \\
0.74 \\
x\end{array}$ & $\begin{array}{c}1.18 \\
x \\
1.18\end{array}$ & $\begin{array}{c}0.61 \\
x \\
0.30 \\
x\end{array}$ & $\begin{array}{l}2.70 \\
1.01 \\
0.33 \\
0.67\end{array}$ & $\begin{array}{c}x \\
x \\
1.08 \\
0.36\end{array}$ & $\begin{array}{c}x \\
x \\
1.94 \\
0.32\end{array}$ & $\begin{array}{c}0.31 \\
x \\
0.94 \\
x\end{array}$ & $\begin{array}{l}1.22 \\
0.61 \\
0.91 \\
2.14\end{array}$ & $\begin{array}{l}0.37 \\
0.32 \\
1.62 \\
0.64\end{array}$ & $\begin{array}{c}0.72 \\
x \\
1.09 \\
3.27\end{array}$ & $\begin{array}{l}1.05 \\
0.70 \\
0.70 \\
2.45\end{array}$ & $\begin{array}{l}2.30 \\
0.30 \\
2.30 \\
7.23\end{array}$ & $\begin{array}{c}3.26 \\
x \\
3.62 \\
10.14\end{array}$ & $\begin{array}{l}.3 .77 \\
2.83 \\
2.83 \\
\mathbf{3 . 7 7}\end{array}$ \\
\hline $\begin{array}{l}\text { Praemurica taurica } \\
\text { P. pseudoinconsians }\end{array}$ & 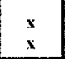 & $\mathrm{x}$ & 0.31 & $\begin{array}{c}0.55 \\
\mathrm{x}\end{array}$ & $\begin{array}{c}0.62 \\
x\end{array}$ & 1.85 & $\begin{array}{c}0.38 \\
x\end{array}$ & $\mathbf{x}$ & $\mathrm{x}$ & $\begin{array}{l}1.01 \\
0.33\end{array}$ & $\begin{array}{c}1.80 \\
x\end{array}$ & $\begin{array}{l}3.89 \\
0.65\end{array}$ & $\frac{2.20}{x}$ & $\begin{array}{c}5.19 \\
x\end{array}$ & $\begin{array}{l}5.82 \\
1.29\end{array}$ & $\begin{array}{l}6.91 \\
1.99\end{array}$ & $\begin{array}{l}5.61 \\
1.75\end{array}$ & $\begin{array}{l}5.92 \\
\mathbf{0 . 3 3}\end{array}$ & $\begin{array}{l}3.26 \\
1.08\end{array}$ & $\begin{array}{l}8.80 \\
2.51\end{array}$ \\
\hline $\begin{array}{l}\text { Chiloguembelina morsei } \\
\text { Ch. midwayensis } \\
\text { Ch. taurica }\end{array}$ & $\begin{array}{l}15.21 \\
2.31 \\
5.94\end{array}$ & $\begin{array}{r}55.48 \\
4.57 \\
4.28\end{array}$ & $\begin{array}{l}56.25 \\
2.81 \\
7.81\end{array}$ & $\begin{array}{l}45.05 \\
3.29 \\
12.36\end{array}$ & $\begin{array}{c}45.34 \\
5.90 \\
9.93\end{array}$ & $\begin{array}{l}+8.30 \\
9.53 \\
9.14\end{array}$ & $\begin{array}{c}39.03 \\
7.80 \\
9.66\end{array}$ & $\begin{array}{c}52.82 \\
3.26 \\
16.61\end{array}$ & $\begin{array}{l}38.58 \\
1.54 \\
9.87\end{array}$ & $\begin{array}{c}31.03 \\
0.67 \\
12.50\end{array}$ & $\begin{array}{c}38.62 \\
7.22 \\
13.71\end{array}$ & $\begin{array}{c}34.41 \\
4.22 \\
10.38\end{array}$ & $\begin{array}{c}33.12 \\
5.04 \\
16.72\end{array}$ & $\begin{array}{l}40.06 \\
6.72 \\
12.53\end{array}$ & $\begin{array}{l}3.3 .98 \\
3.56 \\
13.26\end{array}$ & $\begin{array}{c}54.90 \\
5.81 \\
8.36\end{array}$ & $\begin{array}{c}26.66 \\
4.91 \\
15.08\end{array}$ & $\begin{array}{l}+8.68 \\
2.63 \\
8.22\end{array}$ & $\begin{array}{c}38.04 \\
2.89 \\
5.43\end{array}$ & $\begin{array}{l}23.58 \\
3.46 \\
8.17\end{array}$ \\
\hline $\begin{array}{l}\text { Woodringina clay tonensis } \\
\text { W. hornesstownensis }\end{array}$ & $\begin{array}{c}10.23 \\
4.29\end{array}$ & $\begin{array}{l}2.46 \\
.1 .52\end{array}$ & $\begin{array}{l}4.06 \\
6.87\end{array}$ & $\begin{array}{l}4.67 \\
4.94\end{array}$ & $\begin{array}{l}2.48 \\
5.28\end{array}$ & $\begin{array}{l}1.84 \\
2.46\end{array}$ & $\begin{array}{l}1.48 \\
1.48\end{array}$ & $\begin{array}{l}1.48 \\
1.78\end{array}$ & $\begin{array}{l}1.85 \\
0.92\end{array}$ & $\begin{array}{l}1.01 \\
3.04\end{array}$ & $\begin{array}{l}2.16 \\
3.97\end{array}$ & $\begin{array}{l}1.94 \\
1.94\end{array}$ & $\begin{array}{l}3.78 \\
\mathbf{0 . 6 3}\end{array}$ & $\begin{array}{l}0.91 \\
0.91\end{array}$ & $\begin{array}{l}0.64 \\
1.94\end{array}$ & $\begin{array}{c}x \\
0.72\end{array}$ & $\begin{array}{l}\mathbf{0 . 7 0} \\
\mathbf{0 . 7 0}\end{array}$ & 0.65 & $\begin{array}{l}1.08 \\
1.08\end{array}$ & $\begin{array}{c}x \\
1.25\end{array}$ \\
\hline $\begin{array}{l}\text { Guembelitria danica } \\
\text { G. irregularis } \\
\text { G. alabomensis } \\
\text { G. cretacea } \\
\text { G. irifolia }\end{array}$ & $\begin{array}{l}2.64 \\
5.61 \\
3.25 \\
10.36 \\
3.36\end{array}$ & $\begin{array}{l}10.91 \\
1.76\end{array}$ & $\begin{array}{l}10.62 \\
2.18\end{array}$ & $\begin{array}{l}5.49 \\
5.22\end{array}$ & $\begin{array}{c}2.17 \\
3.41 \\
6.83 \\
X\end{array}$ & $\begin{array}{l}4.30 \\
1.84 \\
6.77 \\
2.46\end{array}$ & $\begin{array}{c}10.03 \\
0.74\end{array}$ & $\begin{array}{l}2.96 \\
\text {..18 }\end{array}$ & $\begin{array}{c}1.23 \\
2.47 \\
0.30 \\
17.39 \\
3.39\end{array}$ & $\begin{array}{l}11.82 \\
8.10\end{array}$ & $\begin{array}{l}9.38 \\
2.52\end{array}$ & $\begin{array}{l}2.98 \\
0.97\end{array}$ & $\begin{array}{c}3.78 \\
x\end{array}$ & $\begin{array}{c}0.61 \\
x \\
0.91\end{array}$ & x & $\begin{array}{l}1.45 \\
0.36\end{array}$ & 0.35 & $\mathbf{0 . 3 3}$ & & \\
\hline $\begin{array}{l}\text { Helerohelix planala } \\
\text { H. globulosa } \\
\text { H. navarroensis }\end{array}$ & $\begin{array}{l}x \\
2.97 \\
2.31\end{array}$ & $\begin{array}{c}x \\
\mathbf{L} .06 \\
2.11\end{array}$ & $\begin{array}{c}x \\
1.25 \\
0.94\end{array}$ & $\begin{array}{l}0.28 \\
2.21 \\
0.83\end{array}$ & $\begin{array}{l}0.31 \\
1.53 \\
0.93\end{array}$ & $\begin{array}{c}x \\
0.62 \\
0.31\end{array}$ & $\begin{array}{l}0.38 \\
0.38\end{array}$ & $\mathrm{x}$ & $\mathrm{x}$ & 0.34 & & & & & & & & & & \\
\hline Glohigerinelloides yaucoensis & $\mathbf{x}$ & 0.35 & & & & & & & & & & & & & & & & & & \\
\hline H. holmdelensis & $x$ & & & & & & & & & & & & & & & & & & & \\
\hline TOTAL NUMBER COUNTED & 303 & 284 & 320 & 362 & 322 & 325 & 266 & 337 & 324 & 296 & 276 & 308 & 317 & 327 & 309 & 275 & 285 & 304 & 276 & 318 \\
\hline
\end{tabular}

\section{BIOSTRATIGRAPHY}

None of the biozonations defined for the $K / T$ transition is completely applicable for this section. The classical biozonation of Bolli (1966) did not have sufficient resolution since the $\mathrm{K} / \mathrm{T}$ boundary remained unzoned at that time. Berggren (1969, 1971 ) and Blow (1979) generalised the use of a biostratigraphical system of numerical nomenclature for the biozones, which has been modified by different authors and the result is quite confusing. The Agost section is very similar to the Spanish section at Caravaca and quite similar to the very well known El Kef section in Tunisia. Our zonation is similar to that of Pardo et al. (1996) for the uppermost Maastrichtian and lowermost Danian, although we prefer to use the classical system of nomenclature, and our biozonation differs as discussed below. Figure 1 shows the datum events at the Agost section along with our biozonation and the comparison with other related biozonations.

\section{Abathomphalus mayaroensis Biozone}

This biozone was originally defined by the total range of $A$. mayaroensis, but in this study following the zonation proposed by Pardo et al. (1966) is restricted to the first appearance datum (FAD) of Plummerita hantkeninoides. The A. mayaroensis Biozone characterise the late Maastrichtian in low latitude and high latitude deep sea sequences such as the Tethyan palaeogeographic realm (Robaszynski 


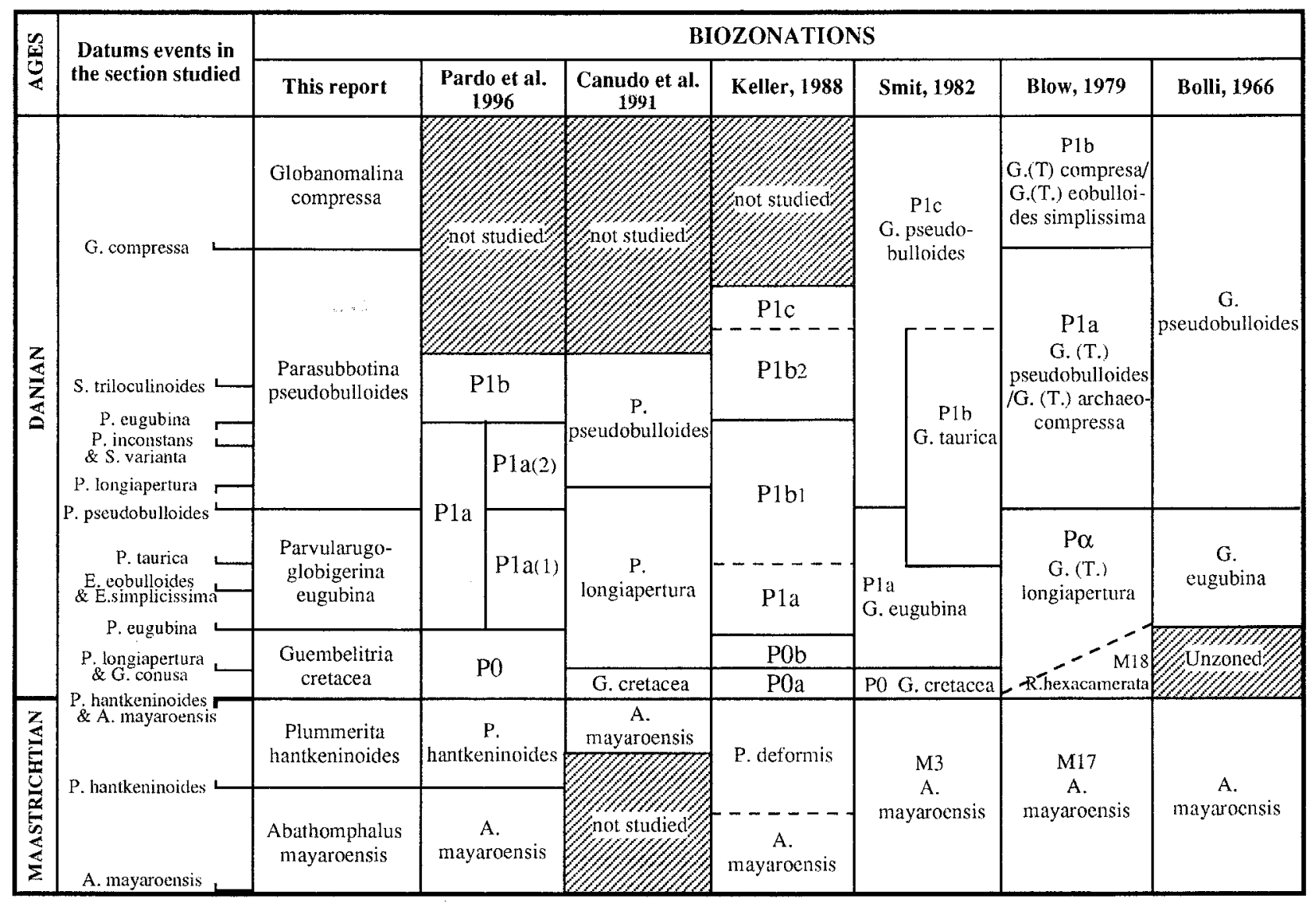

FIG. 1. - Comparison of the most relevant planktic foraminiferal biozonations across the K/T boundary.

Comparaison des biozonations de Foraminifères planctoniques les plus significatives à travers la limite K/T.

et al., 1983-1984), including the Alamedilla (Linares, 1977) and Caravaca sections (Canudo et al., 1991) and the southern Indian Ocean Sites 690 and 738 (Huber, 1991; Keller, 1993). However, A. mayaroensis is very rare or absent in shallow sections such as El Kef (Keller, 1988), Brazos River (Keller, 1989a, b) and Stevns Klint and Nye Klov in Denmark (Schmitz et al., 1992; Keller et al., 1993). This biozone is thus restricted mainly because $A$. mayaroensis is a deep water dweller. Therefore, alternative biozones have been proposed to characterise the terminal Maastrichtian. The Globutruncana falsocalcarata Biozone was originally proposed by Kassab (1976) based on sections in northern Iraq and applied by Solakius et al. (1984) in the Ain Mdeker section in Tunisia. However, few workers have observed the first appearance of the index species prior to the FAD of $A$. mayaroensis. The Pseudotextularia deformis Biozone was propo- sed by Keller (1988) but this index species has taxonomical problems.

\section{Plummerita hantkeninoides Biozone}

This biozone is defined by the total range of Plummerita hantkeninoides and has been recognised by Pardo et al. (1996) at the Agost section. Nevertheless, Masters (1993) had previously suggested that $P$. hantkeninoides is a junior synonym of Rugoglobigerina reicheli, but we think that both morphotypes belong to different species. It is used in this paper due to the scarcity of $A$. mayaroensis in the terminal Maastrichtian mainly in shallow sections as indicated above. Nevertheless, in the two uppermost Cretaceous samples $A$. mayaroensis is more frequent that $P$. hantkeninoides. The abundance of $A$. mayaroensis just below the layer with the iridium anomaly has also been observed in some 
continuous sections in the Basque Country in Northern Spain (Orúe-Etxebarría et al., 1991; Orúe-Etxebarría, com. pers.). The presence of $A$. mayaroensis just below the $\mathrm{K} / \mathrm{T}$ boundary in these sections could be associated with a sea-level rise during the last $50 \mathrm{kyr}$ of the Maastrichtian ( $\mathrm{Li}$ and Keller, in press). All large tropical Cretaceous taxa suddenly disappear at the top of this biozone. At the Agost section, the total range of $P$. hantkeninoides spans the top $3.45 \mathrm{~m}$ of the Maastrichtian, whereas at the El Kef stratotype it corresponds to the top $6 \mathrm{~m}$. This suggest that the El Kef section has a higher rate of sedimentation than the Agost section.

\section{Guembelitria cretacea Biozone}

This biozone spans the interval between the last appearance datum (LAD) of Plummerita hantkeninoides precisely at the K/T boundary and the first appearance datum of $G$. eugubina. The $G$. cretacea Biozone is the equivalent to Zone P0 of Smit (1982), Keller (1988) and Pardo et al. (1996). The K/T boundary was recently defined at the base of a clay layer at the El Kef boundary stratotype section, where the $K / T$ boundary is recognised by a thin red oxidized layer, an Ir anomaly, an increase in Ni-rich spinels, an increase in total organic carbon (TOC), a decrease in $\partial^{13} \mathrm{C}$, and the first appearance of the Tertiary planktic foraminifera. This boundary clay and its basal red layer is easily correlated with the basal yellow-red layer of Jarosite and $\mathrm{FeO}$, which is just below an interval of 10 centimetres thick dark clay at the Agost section. This yellow-red layer contains geochemical evidence that is related to the effect of a large meteorite impact (Smit and ten Kate; 1982, Smit, 1990). Most of the $G$. cretacea Biozone is characterised by black clay which contains a well preserved autochthonous fauna and the Tertiary planktic foraminifera first appeared in the lower part of this biozone.

\section{Parvularugoglobigerina eugubina Biozone}

This biozone defines the interval between the $P$. eugubina FAD and the $P$. pseudobulloides LAD. Keller (1993) defines the $P$. eugubina Biozone as the total range of this species and uses the $P$. pseudobulloides FAD to subdivide this biozone into Pla(1) and Pla(2). Canudo et al.(1991) follow Blow (1979) and used P. longiapertura to characterise this biozone because this species has a very distinct and stable morphology with its compressed test and high slit-like aperture and thus may be a more suitable index taxon. Nevertheless, to date this alternative biozone has been less accepted and most workers have included $P$. longiapertura within the $P$. eugubina group. For this reason we decided to continue the use of the classical $P$. eugubina Biozone.

\section{Parasubbotina pseudobulloides Biozone}

This biozone defines the interval between the $P$. pseudobulloides FAD and Globanomalina compressa FAD. Parasubbotina pseudobulloides is an index taxon that has been used in most of the classical biozonations. Nevertheless, the FAD of $S$. pseudobulloides appears to be very diachronous (MacLeod and Keller, 1991 ab) and Canudo et al. (1991) suggested the $P$. longiapertura LAD as alternate datum event. However, this proposal also has taxonomic and biostratigraphic problems and we decided to follow the standard biozonation of Bolli (1966). In the lower part of this zone, planktic foraminifera reach normal size and can be found in the fraction larger than 150 microns, as also observed by Keller (1988) for the El Kef section.

\section{Globanomalina compressa Biozone}

This biozone defines the interval between the $G$. compressa FAD and the top of the section studied. This event was used by Blow (1979) to define the base of the G.(T.) compressa/G(T.) eobulloides simplicissima (P1 b) Biozone. This interval is well above the $K / T$ boundary and not relevant for the $K / T$ boundary event, since all but one Cretaceous taxa (G. cretacea) became extinct earlier, and the adaptive radiation of early Tertiary taxa is well established.

\section{FAUNAL TURNOVER}

Late Maastrichtian assemblages from the Agost section are largely dominated by biserial species (heterohelicids). Planispiral (globigerinelloids) and trochospiral (hedbergellids, rugoglobigerinids and globotruncanids) species are frequent, triserial species (guembelitriids) are common and tubulospinose (schackoinids) species are rare. The stratigraphic ranges of these taxa during the late Maastrichtian indicate various changes in the faunal assemblages but most of species are present in the $A$. mayaroensis and P. hantkeninoides Biozones. Environ- 
mental changes are indicated by a sequence of species extinctions that begin at the base of the upper Maastrichtian (base of $A$. mayaroensis Biozone) and continue up to the $K / T$ boundary. The first species to disappear are Gansserina wiedenmayeri, Gansserina gansseri and Contusotruncana plicata in the A. mayaronensis Biozone. The rest of the species (Racemiguembelina milamensis, Archaeoglobigerina cretacea, Gublerina acuta and $\mathbf{R u}$ goglobigerina pennyi) disappear in the upper part of the P. hantkeninoides Biozone. Only one species has its first appearance in this interval : $P$. hantkeninoides. Therefore, more species disappear than appear in the late Maastrichtian and this tendency is more evident in the uppermost metre where 4 , species went extinct and no species originated (Fig. 2). This suggests adverse environmental conditions during the latest Maastrichtian.

A total of 47 species disappear in coincidence with the yellow-red layer that marks the $\mathrm{K} / \mathrm{T}$ boundary. We double checked this coincidence by scanning the residue of the uppermost Cretaceous samples. This methodology is necessary in order to avoid the Signor-Lipps (1982) effect, because certain rare species could appear to become extinct before their real moment of extinction. The large, complex, tropical and deep dwelling forms are less frequent in the terminal Cretaceous, indicating that these latitudinal restricted species were declining (Fig. 3).

In the Agost section there is almost no evidence of reworking, the sedimentology shows no evidence of currents or other indications of bioturbation. To eliminate the potential problem of reworking, that could interfere in high resolution sampling, we ignored the presence of isolated specimens in a sample, assuming them to be reworked and hence did not list them in our data tables or figures. In the lowermost Tertiary (G. cretacea, P. eugubina, $P$. pseudobulloides and the lower part of $G$. compressa Biozones) a total of 16 Cretaceous species are present that can be considered Cretaceous survivors. The 16 Cretaceous survivors are cosmopolitan small, simple surface dwellers that disappeared gradually during the early Danian (Fig.4). Survivorship of cosmopolitan Cretaceous species was first noted by Keller $(1988,1989 a, b)$ and later documented in numerous sections worldwide (Canudo et al.,
1991 ; Keller et al., 1993 ; MacLeod and Keller, 1994).

In the same Danian levels in which the surviving Cretaceous species are disappearing, new Tertiary species are evolving. These new species are also opportunistic cosmopolitan taxa which can be divided into two main assemblages. The first assemblage to appear consists of the small cosmopolitan surface dwellers which originate in the $G$. cretacea Biozone and become extinct in the lower part of the $P$. pseudobulloides Biozone. The second assemblage originates in the $P$. eugubina Biozone and diversifies during the lower part of the $P$. pseudobulloides Biozone where these taxa reach normal size. This assemblage colonises the intermediate and deeper water environments in the upper part of this biozone and they continue to be abundant at least to the top of the interval studied (Fig. 5).

Quantitative planktic foraminiferal analysis of the uppermost $2.25 \mathrm{~m}$ of the Cretaceous shows little variation among the relative abundances of the different species (Fig. 3). The total percentage of the species that became extinct at or below the K/T boundary is about $20 \%$ in the size fraction larger than 63 microns. All of these taxa are large, complex forms adapted to deep environments. In contrast, the small, cosmopolitan forms which were largely surface dwellers were most abundant (e.g., Heterohelix globulosa and Heterohelix navarroensis). The guembelitriids (Guembelitria trifolia and G. cretacea) are rare in the upper Cretaceous, but these opportunistic species are very abundant in the lowermost Tertiary, just after the main planktic foraminiferal extinction event. The rest of the Cretaceous surface dwellers are less abundant in the Danian than in the Maastrichtian (Fig. 3).

The relative abundance of species that evolved in the lower Danian are dominant during the early Danian and most of them became extinct near the base of the $P$. pseudobulloides Biozone (e.g., Parvularugoglobigerina longiapertura, $P$. eugubina, $P$. sabina, Globoconusa fodina and Globoconusa conu$s a)$. After the disappearance of these species, the dominant species are Woodringina hornerstownensis, Woodringina claytonensis, Chiloguembelina morsei, Chiloguembelina taurica and Chiloguembelina midwayensis (Fig. 5).

FIG. 2. - Species ranges of upper Maastrichtian planktic foraminifera at Agost.

Distribution stratigraphique des Foraminifêres planctoniques au Maastrichtien supérieur à Agost. 


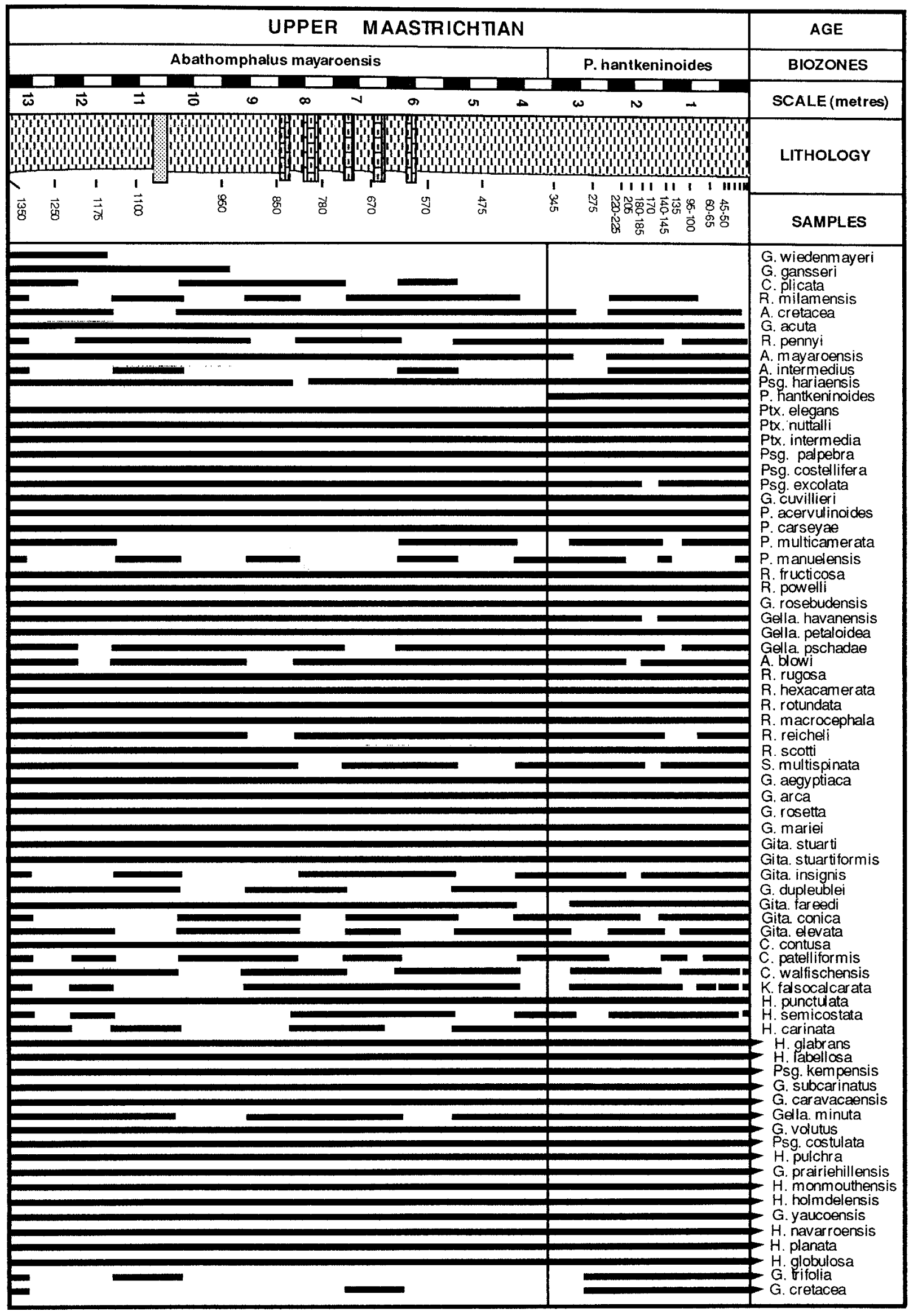




\section{MODELS AND CAUSES OF EXTINGTION}

The planktic foraminiferal extinction model for the K/T boundary is a controversial topic because it depends on different interpretations of the data and on the methodology used to obtain these data (Molina, 1994). The continuity of the Agost section, the abundance of planktic foraminifera, the high resolution sampling and the quantitative study provide an excellent opportunity to evaluate the $\mathrm{K} / \mathrm{T}$ boundary mass extinction and elucidate the real nature of this controversy.

The oldest and least detailed studies of the K/T transition were performed on widely spaced samples (Bolli, 1966) or on sections which contained hiatuses (Linares and Martínez-Gallego, 1971). At present it is quite obvious that such methodology, or discontinuous sections are unsuitable to establish the pattern of extinction in planktic foraminifera, and that by default such studies will show a catastrophic extinction pattern. An additional constraint in evaluating this mass extinction is the thickness of the K/T transition, or the rate of sediment deposition. Luterbacher and Premoli-Silva (1964) sampled and studied Gubbio section in great detail. They defined several new species and named a new biozone ( $G$. eugubina Biozone). However, this Italian section is very condensed and contains a hiatus (MacLeod and Keller, 1991a,b) and thus can not be used as the real model of extinction. Alvarez et al. (1980) proposed the bolide impact hypothesis for the Cretaceous-Tertiary mass extinction based on these Italian sections, and hence assumed the nearly total extinction of the planktic foraminifera. This concept of near total extinction was the prevailing view among micropaleontologists at that time.

Nevertheless, more expanded sections were studied by Smit $(1977,1979)$ at that time who established the new $G$. cretacea Biozone based on the Caravaca section (Spain). But he also assumed the total extinction of all the planktic foraminifera, except $G$. cretacea and probably $H$. monmouthensis and $G$. messinae. Although Smit studied closely spaced samples across the $\mathrm{K} / \mathrm{T}$ boundary, he concluded that the extinction of planktic foraminifera was abrupt and without any previous warning in the sedimentary record (Smit and Hertogen, 1980). Smit also studied the Spanish section at Agost (Groot et $a l ., 1989$ ) and the Tunisian section at El Kef, and maintained and even further developed the idea that the impact event at the K/T boundary suddenly exterminated all but one species (Smit, 1982, 1990). In order to maintain this interpretation, he considered all Cretaceous specimens present above the $K / T$ boundary as reworked. These data were not questioned until Keller (1988) also studied the El Kef section and provided evidence that species extinction extended across the $\mathrm{K} / \mathrm{T}$ boundary with 10 of the species surviving well into the Tertiary. She also found 14 species disappearing below the boundary and concluded that these extinctions appear unrelated to an impact event. These ideas were also documented studying other sections distributed all over the world (Keller, 1989a,b, 1990, 1994). Nevertheless, some authors critically commented those ideas (Huber et al., 1994), which were well defended and largely replied (Keller and MacLeod, 1994).

One of the most controversial aspect in the K/T extinction pattern is the decline of the fauna prior to the K/T boundary. Our data from Agost indicate that 7 species disappeared but just one evolved during the late Maastrichtian. This decline is most apparent in the terminal Maastrichtian (uppermost meter) where 4 species disappeared, but no species appeared (Fig. 2). The quantitative data show that other large species decreased in abundance and are rare in the terminal Cretaceous (Table 1, Fig. 2 and 3 ). Changes prior to the $\mathrm{K} / \mathrm{T}$ boundary were also noted in the Spanish sections of Zumaya (Herm, 1965 ; Lamolda, 1983 ; 1990), Sopelana (Lamolda et $a l ., 1983$; Orúe-Etxebarría et al., 1984), Caravaca (Canudo et al., 1991 ; Canudo 1994; Pardo et $a l ., 1996$ ) and in the Tunisian section of El Kef (Brinkhuis and Zachariasse, 1988). However, the magnitude of the decline shown by the former authors is less notable than the one found at $\mathrm{El}$ Kef (Keller, 1988, $\mathrm{Li}$ and Keller, in press), at Brazos River (Keller, 1989 a,b) and in the Antarctic Ocean (Keller, 1993).

The differences among the patterns of extinction found in all of these sections are primarily due to latitudinal effects, with large specialized forms disappearing first from high latitudes. This indicates that the decline prior to the $\mathrm{K} / \mathrm{T}$ boundary is mainly due to a gradual decrease in temperature (Schmitz et al., 1992 ; Keller et al., 1993 ; Barrera, 1994;

FIG. 3. - Relative abundances of typical Maastrichtian species at Agost.

Abondance relative des espèces typiques du Maastrichtien à Agost. 


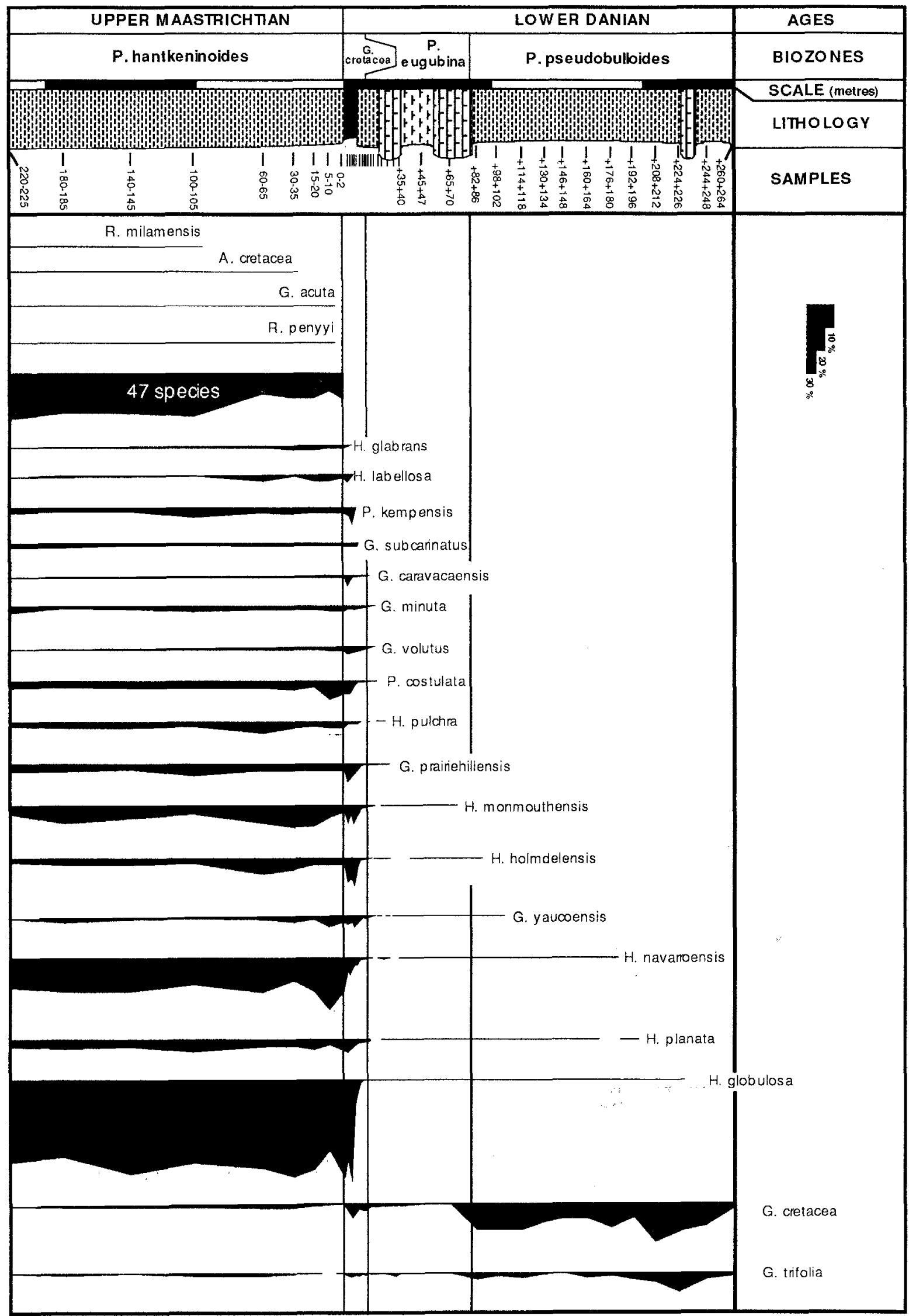




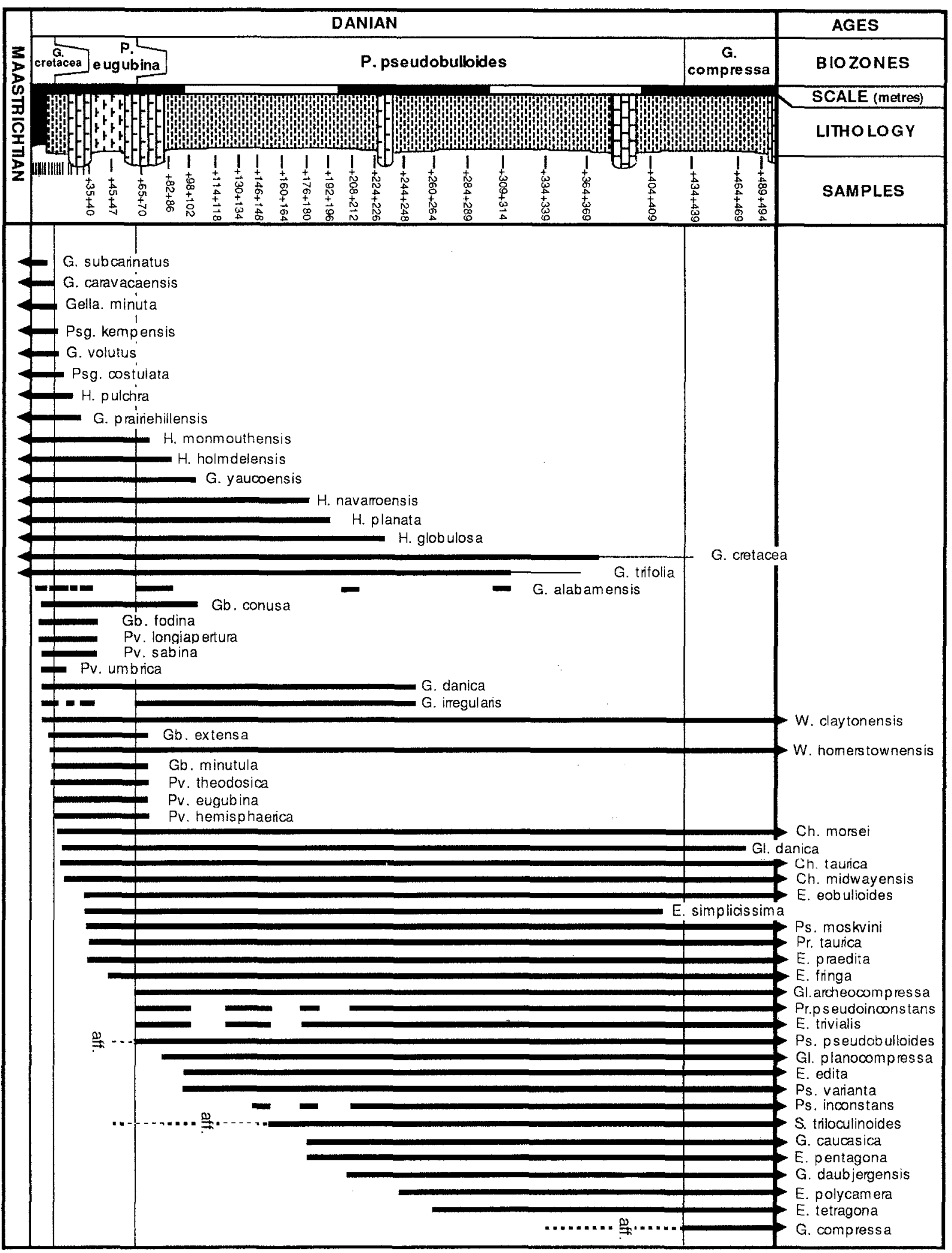

FIG. 4. - Species ranges of Danian planktic foraminifera at Agost.

Distribution stratigraphique des Foraminiferes planctoniques au Danien à Agost. 
D'Hondt and Lindinger, 1994), which produced the retreat to low latitudes of certain species. However, the discrepancies in interpretations in low latitudes and even in the same sections, are due to different taxonomic interpretations and the Signor-Lipps effect. Nevertheless, the Signor-Lipps effect does not explain the absence of extinctions prior the $K / T$ boundary (Smit, 1982, 1990) and can not be invoked to conclude that there were no significant plankton extinetions before the K/T boundary (Smit, 1994). According to our data from Agost the extinctions prior to the $\mathrm{K} / \mathrm{T}$ boundary are significant, although they seem not to be very important in magnitude because species affected were already rare and retreating from high latitudes to refuge in low latitudes. The extinetion of $G$. wiedenmayeri, $G$. gansseri and $C$. plicata in the $A$. mayaroensis Biozone could be considered as the result of background extinction, but the extinction of $R$. milamensis, A. cretacea, G. acuta and R. pennyi in the $P$. hantkeninoides Biozone are very probably part of a gradual mass extinction that ended in the $P$. pseudobulloides Biozone.

In order to establish the magnitude of the extinction, which precisely coincides with the layer that characterises the $K / T$ boundary, suitable sections and the right methodology are necessary. The sections have to be continuous, well expanded, not very shallow and located in low latitudes where the most diversified faunas lived. These qualifications apply to the Agost section which is very continuous since all the planktic biozones have been found. This section is similar to El Kef and Caravaca which are the most complete sections known world wide (MacLeod and Keller, 1991 a, b). The paleoenvironment at Agost was a subtropical upper slope, based on small benthic foraminifera (Pardo et al., 1996), with a very diversified planktic foraminifera fauna. The high resolution methodology and the intensive search in every terminal Cretaceous sample (to avoid the Signor-Lipps effect) indicate that 47 species suddenly disappeared at the $\mathrm{K} / \mathrm{T}$ boundary at Agost section (Fig. 2, 3 and 6). Although this extinction event represents only about $20 \%$ of the individuals in the population in the fraction larger than 63 microns, it is the major extinction event in the history of planktic foraminifera, and it exactly coincides with the layer containing the impact evidence. Regarding the causes of this major extinction, now it is generally accepted that a meteorite impacted at the $\mathrm{K} / \mathrm{T}$ boundary (D'Hontd, 1994; Schmitz, 1994). The species that suddenly became extinct were the deep dwellers and the majority of the intermediate dwellers, whereas the cosmopolitan surface dwellers suffered less, the very probable reason being that they were protected in high latitudes, recolonizing the low latitudes after the impact event.

How many species survived the $\mathrm{K} / \mathrm{T}$ boundary event? This question constitutes one of the most controversial aspects of this topic. According to Smit $(1982,1990)$ only Guembelitria cretacea survived. In contrast, Keller $(1988,1989$ a, b, 1993, 1994), Keller et al. (1993) and MacLeod and Keller (1994) reported that about $1 / 3$ of the species survived. Historically it was assumed by most of the micropaleontologists that almost all planktic foraminifera became extinct at the $\mathrm{K} / \mathrm{T}$ boundary; consequently, all Cretaceous specimens found in the basal Danian were considered as reworked. However, isotopic analyses of some Cretaceous species present in earliest Tertiary sediments allowed Barrera and Keller, (1990) and Keller et al. (1993) to conclude that most of the abundant Cretaceous species present in Tertiary sediments have Tertiary signals and are therefore Cretaceous survivors. Most of the 16 species found in the basal Tertiary of the Agost section can be considered survivors on the base of their constant presence in several samples and their geographic distribution in other sections (Keller, 1988, 1989a,b, 1993 ; Canudo et $a l ., 1991$; Keller et al., 1993 ; MacLeod and Keller, 1994). Nevertheless, not all Cretaceous specimens present in earliest Tertiary samples are survivors; for instance, in samples from the basal $3 \mathrm{~cm}$ of the G. cretacea Biozone we found some globotruncanids, and in samples up section, we found isolated specimens with different preservation, which we considered reworked. Most of the Cretaceous species frequent and present in several Tertiary samples are considered as autochthonous. However, Heterohelix glabrans and Heterolelix labellosa that are frequent only in the basal samples of the Tertiary may be reworked and need further confirmation in other sections. Lately, certain foraminiferal specialists that have criticised the Cretaceous species survivorship data, are now accepting that some species, such as Hedbergella holmdelensis and Hedbergella monmouthensis, survived apart from $G$. cretacea (Liu and Olsson, 1994). Even Smit (1995) now admits some, "unimportant", Cretaceous species survivors above the $K / T$ boundary and that "the final extinctions may have lingered on for a while". At the same time that the Cretaceous survivors are gradually disappearing in the basal Danian, other small species are gradually originating. The absence of certain primitive species in the lower part of $G$. cretacea Biozone could be due to carbonate dissolution in this deep sea section or to the delay in the recolonization of this latitude (Fig. 6). 
The Tertiary species ecologically replaced the Cretaceous species that could not successfully adapt to new environmental conditions (MacLeod, 1993). By the P. pseudobulloides Biozone, this adaptive radiation began to colonise the vacant deep environments and species morphology reached again normal size for planktic foraminifera. This gradual pattern of extinction and origination along the $\mathrm{K} / \mathrm{T}$ transition is difficult to explain by the impact theory. The gradual pattern of extinction seems to be mainly controlled by geological causes (volcanism, temperature changes, ocean circulation changes, sea level changes) and although biological causes (competition, endemism) could be subordinated, they would play an important role (Molina, 1995). The gradual pattern is plausibly explained by a decrease in temperature that began in the uppermost Cretaceous (Schmitz et al., 1992; Barrera, 1994; D'Hondt and Lindinger, 1994). The decrease in temperature could probably be associated with a sea level change (Ginsburg, 1964, 1984, Brinkhuis and Zachariasse, 1988; Keller, 1988). This gradual pattern is also consistent with the effect of massive volcanism (Officer y Drake, 1985 ; Courtillot et al., 1986) that lasted at least 3 million years beginning about 2 million years before the K/T boundary (Venkatesan et al., 1993). Consequently, the probable cause that produced the gradual mass extinction of 23 species during the upper Maastrichtian and lower Danian, is not as simple as an extraterrestrial impact that produced the catastrophic mass extinction of the 47 species at the $K / T$ boundary.

\section{CONCLUSIONS}

The section of Agost in Spain is one of the most continuous and expanded marine record of the $K / T$ transition known so far. The planktic foraminiferal assemblages are very rich, diversified and well preserved. Six biozones have been recognised across the K/T boundary: Abathomphalus mayaroensis Biozone and Plummerita hantkeninoides Biozone (late Maastrichtian), Guembelitria cretacea Biozone, Parvularugoglobigerina eugubina Biozone, Para- subbotina pseudobulloides Biozone and Globanomalina compressa Biozone (early Danian). These biozones have been correlated with other biozonations established in different geographical regions.

The quantitative study and the high resolution sampling allow us to test the model of extinction in planktic foraminifera across the K/T boundary. The mass extinction occurred over an extended time period similar to the Tunisian stratotype section of El Kef and the Spanish section of Caravaca. At Agost, 7 species disappeared in the late Maastrichtian, 47 became extinct precisely at the $\mathrm{K} / \mathrm{T}$ boundary and 16 disappeared in the earliest Tertiary. The species that became extinct at the $\mathrm{K} / \mathrm{T}$ boundary constitute only about $20 \%$ of the individuals in the population larger than 63 microns. The 4.7 species that suddenly became extinct are large, complex tropical-subtropical forms that lived in deeper to intermediate waters. Their extinction at the $\mathrm{K} / \mathrm{T}$ boundary constitutes the major and catastrophic mass extinction in the history of planktic foraminifera. This pattern of extinction is superimposed onto a gradual pattern of extinctions of 23 species that began in the $A$. mayaroensis Biozone (late Maastrichtian) and ended in the lower part of the $P$. pseudobulloides Biozone (early Danian). Cretaceous species which disappeared gradually are all simple, small forms, of cosmopolitan distribution that lived in surface waters. This group constitutes about $80 \%$ of the individuals in the populations and their disappearance over the latest Maastrichtian to early Danian can be considered a gradual mass extinction.

Our data provide an excellent opportunity to elucidate the ongoing controversy between Smit and Keller, as to whether the $K / T$ mass extinction was catastrophic or gradual. Our mass extinction model in planktic foraminifera is consistent with both patterns, since our data show that a major catastrophic mass extinction that centred at the $\mathrm{K} / \mathrm{T}$ boundary was superimposed on a gradual mass extinction which began in the late Maastrichtian and continued into the early Danian. Our interpretations regarding the causes of the mass extinction in planktic foraminifera are mainly based on the two superimposed patterns we observed and we acknowledge the need for further multidisciplinary studies. The catastrophic mass extinction of 4.7

FIG. 5. - Relative abundances of Danian species at Agost.

Abondance relative des espèces du Danien à Agost. 


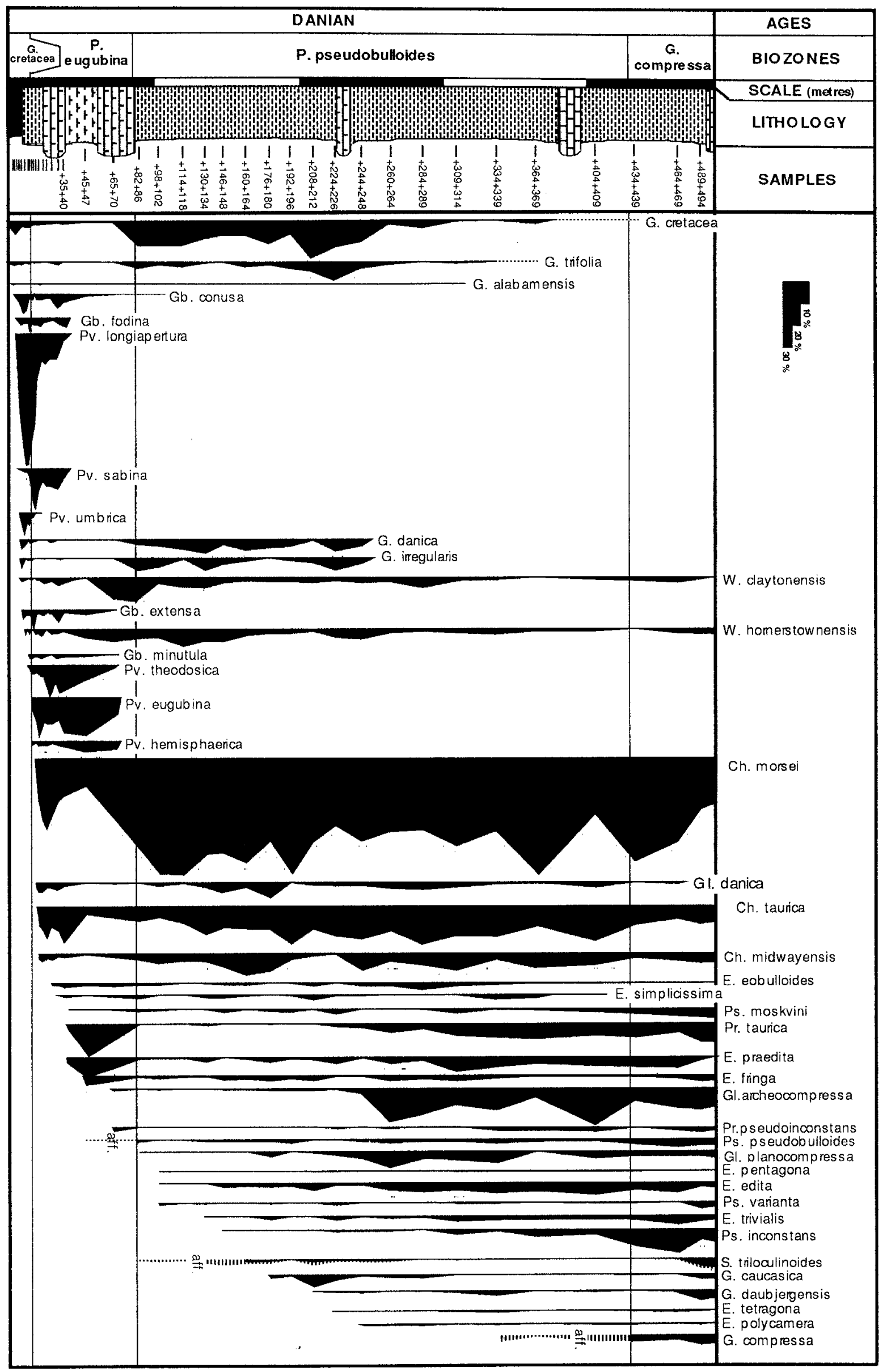




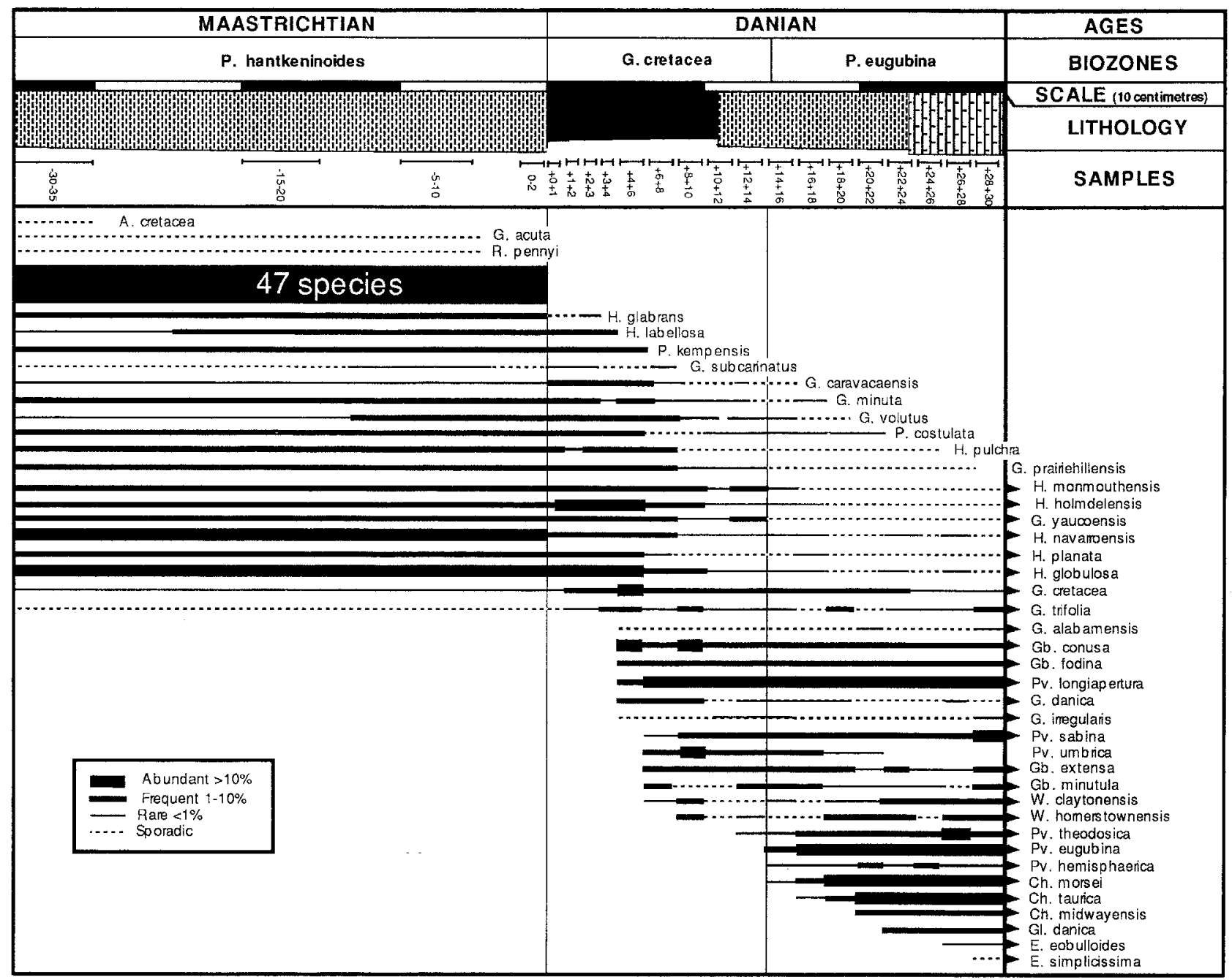

FIC. 6. - Semiquantitative species reanges across the K/T boundary at Agost.

Distribution stratigraphique semi-quantitative des espèces à la limite K/T à Agost.

species at the $\mathrm{K} / \mathrm{T}$ boundary is very compatible with the sudden effects of a large meteorite impact, whereas the gradual mass extinction of 23 species during the Maastrichtian-Danian transition is compatible with temperature and sea level changes that may be related to massive volcanism. Thus, the $K / T$ boundary mass extinction in planktic foraminifera is a more complex phenomenon than that of either a bolide impact or environmental changes alone.

\section{PLATE 1}

1-3. Abathomphalus mayaroensis (BOLL1), sample Agost, 0-2 cm. 4-6. Plummerita hantkeninoides (BrönnimanN), sample Agost, $15-20 \mathrm{~cm}$.

7. Guembelitria cretacea (CUSIIMAN), sample Agost, $82+86 \mathrm{~cm}$.
8-9. Parvularugoblobigerina eugubina (LuTERBacher and PreMoLi SILVA), sample Agost, $28+26 \mathrm{~cm}$.

10-11. Parasubbotina pseudobulloides (Plummer), sample Agost, $260+264 \mathrm{~cm}$. 

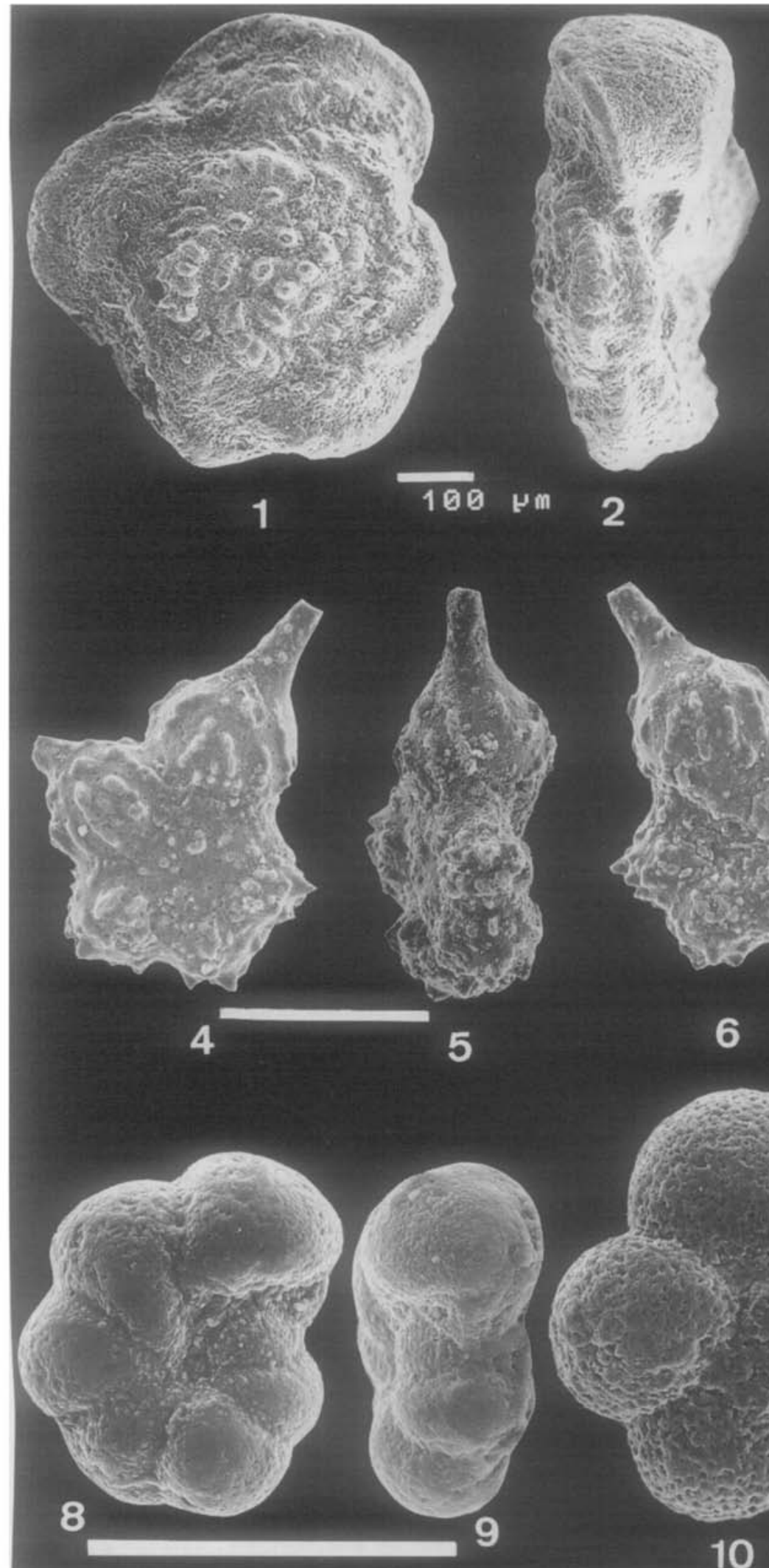

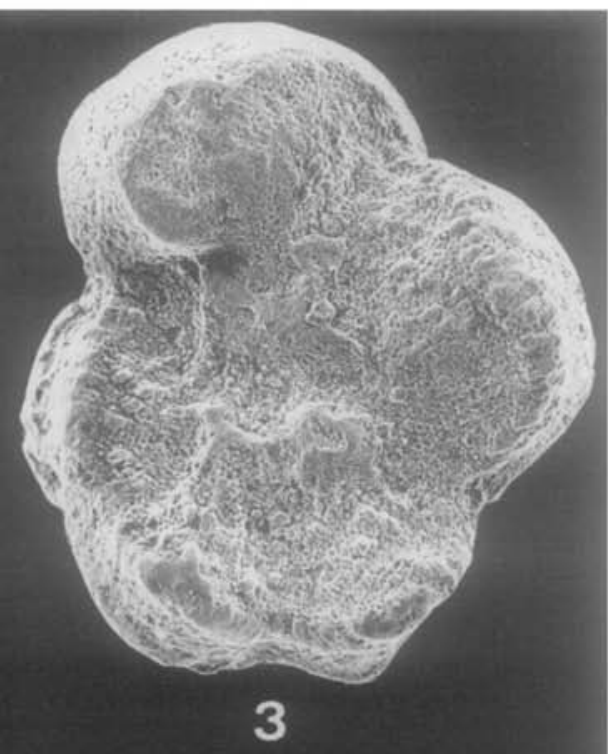

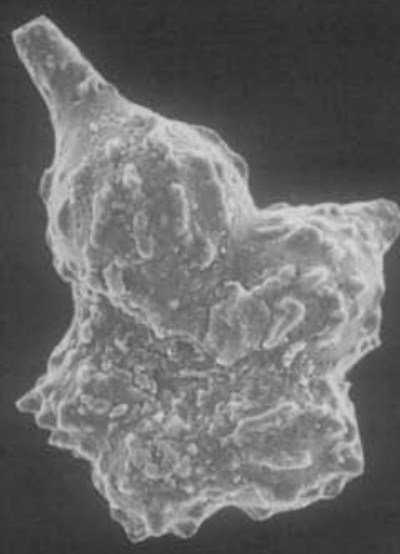

6

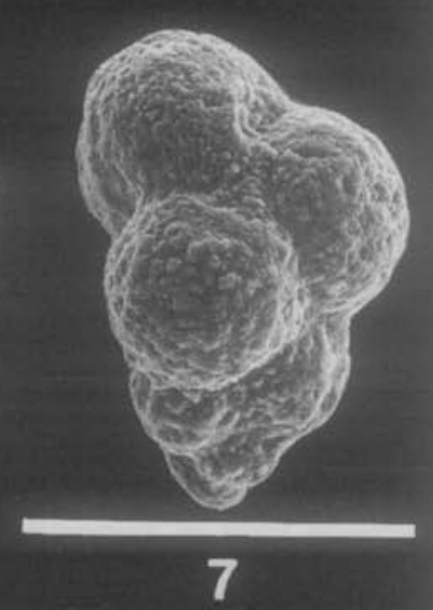




\section{ACKNOWLEDGEMENTS}

This research was funded by DGICYT project PB94-0566, by Diputación General de Aragón grant BCB3692 and by Gobierno de Navarra grant OF/478/92.

\section{BIBLIOGRAPHY}

alvarez L.W., Alvarez W., Asaro F. and Michel H.V. (1980) : Extraterrestrial cause for the Cretaceous-Tertiary extinction. Science, Washington, vol. 208, p. 1095-1108.

ArZ J.A., Canudo J.I. and Motina E. (1992) : Estudio comparativo del Maastrichtiense de Zumaya (Pirineos) y Agost (Béticas) basado en el análisis cuantitativo de los foraminíferos planctónicos. Actas III Cong. Geol. Esp., Salamanca, vol. 1, p. 487-491.

BARRERA E. (1994): Global environmental changes preceding the Cretaceous-Tertiary boundary : Early-late Maastrichtian transition. Geology, Boulder, vol. 22, p. 877-880.

Barrera E. and Keller G. (1990) : Stable isotope evidence for gradual environmental changes and species survivorship across the Cretaceous/Tertiary boundary. Paleoceanography, Washington, vol. 5(6), p. 867-890.

BERGGREN W.A. (1969): Rates of evolution in some Cenozoic planktonic foraminifera. Micropaleontology, New York, vol. 15(3), p. 351-365.

BERGGREN W.A. (1971) : Multiple phylogenetic zonations of the Cenozoic based on planktonic foraminifera. In A. Farinacei (Editor), Proc. II Plank. Conf., Roma, Ed. Tecnoscienza, p. 41-56.

BLow W.H. (1979) : The Cainozoie Globigerinida. Ed. E.J. Brill, Leiden, p. 1-1413.

BoLLI H.M. (1966) : Zonation of Cretaceous to Pliocene marine sediments based on Planktonic foraminifera. Asoc. Venez. Geol. Miner. Petrol., Caracas, vol. 9(1), p. 3-32.

BrinkHUIS H. and ZaCharJasse W.J. (1988) : Dinoflagellate cysts, sea level changes and planktonic foraminifers across the Cretaceous-Tertiary boundary at EI Haria, Northwest Tunisia. Mar. Micropaleontol., Amsterdam, vol. 13, p. 153-191.

C.ANUdo J.I. (1994) : Bioestratigrafía y evolución de los foraminíferos planctónicos en el tránsito Cretácico-Terciario en España. In E. Molina (Editor), Extínción y registro fósil. Extinction and the fossil record. SIUZ Cuadernos Interdisciplinares, Zaragoza, vol. 5, p. 140-164.

CaNudo J.I. and MoLINA E. (1992) : Bioestratigrafía y evolución de los foraminíferos planctónicos del límite Cretácico/Terciario en Osinaga (Pirineo de Navarra). III Cong. Geol. Esp., Salamanca, vol. 2 , p. 54-62.

Canudo J.I., Keller G. and Molina E. (1991) : Cretaceous/Tertiary boundary extinction pattern and faunal turnover at Agost and Caravaca, S.E. Spain. Mar. Micropaleontol., Amsterdam, vol. 17, p. 319-341.

Courtillot V.E., Besse J., Vandamue D., Montigny R., Jaeger J.J, and CAPPETTA H. (1986): Decean flood basalts at the Cretaceous/Tertiary boundary. Earth Plan. Sci. Lett., Amsterdam, vol. 80, p. 361-374.

D'HondT S. (1994): The evidence for a meteorite impact at the Cretaceous/Tertiary boundary. In E. Molina (Editor),
Extinción y registro fosil. Extinction and the fossil record. SIUZ Cuadernos Interdisciplinares. Zaragoza, vol. 5, p. 75 95.

D'Hondt S. and LuNDinger M. (1994) : A stable isotopic record of the Maastrichtian ocean-climate system : South Atlantic DSDP site 528. Palaeogeogr., Palaeoclimatol., Palaeoecol., Amsterdam, vol. 112, p. 363-378.

Ginsburg L. (1964) : Les régressions marines et le problème du renouvellement des faunes au cours des temps géologiques. Bull. Soc. géol. France, Paris, vol. 6(1), p. 13-22.

Ginsburg L. (1984): Théories scientifiques et extinctions des dinosaures. C. R. Acad. Sci. Paris, vol. 298(II), p. 317-320.

Groot J.J., De Jonge R.B.G., LANGereis C.G., Ten Kate W.G.H.Z. and SMIT J. (1989): Magnetostratigraphy of the Cretaceous-Tertiary boundary at Agost (Spain). Earth Plan. Sci. Lett., Amsterdam, vol. 94, p. 385-397.

Herm D. (1965) : Mikropaleontologische-stratigraphische Untersuchungen im Kreideflysch zwischen Deva un Zumaya (Prov. Guipúzcoa, Nordspanien). Zeit. Deutsch. Geol. Ges., Hannover, vol. 115 , p. 277-348.

HUBER B.T. (1971) : Maestrichtian planktonic foraminifer biostratigraphy and the Cretaceous/Tertiary boundary at hole 738C (Kerguelen plateau, Southern indian Ocean). Proc. ODP. Scient. Results, La Joya, vol. 119, p. 451-465.

Huber B.T., LTU C., OLsson R. and Berggren W. (1994) : Comment on "The cretaceous-Tertiary boundary transition in the Antarctic Ocean and its global inplications" by G. Keller. Mar. Micropaleontol., Amsterdam, vol. 24, p. $91-99$.

Kassab I.M. (1976) : Some upper Cretaceous planktonic foraminiferal genera from norhtern Iraq. Micropaleontology, New York, vol. 22(2), p. 215-238.

KELLER G. (1988) : Extinction, survivorship and evolution of planktic foraminifers across the Cretaceous/Tertiary boundary at El Kef, Tunisia. Mar. Micropaleontol., Amsterdam, vol. 13, p. $239-263$.

KELLER G. (1989a) : Extended period of extinctions across the Cretaceous/Tertiary boundary in planktonic foraminifera of continental shelf sections: Implications for impact and volcanism theories. Geol. Soc. Amer. Bull., Boulder, vol. 101 , p. 1408-1419.

KeLLER G. (1989b) : Extended Cretaceous / Tertiary boundary extinctions and delayed population change in planktonic foraminiferal faunas from Brazos River, Texas. Paleoceanography, Washington, vol. 4, p. 287-332.

KeLLen G. (1993): The Cretaceous/Tertiary boundary transitions in the Antarctic Ocean and its global implications. Mar. Micropaleont., Amsterdam, vol. 21, p. 1-45.

KeLLer G. (1994) : Mass extinction and evolution patterns across the Cretaceous-Tertiary boundary. In E. Molina (Editor), Extinción y registro fósil. Extinction and the fossil record. SIUZ Cuadernos Interdisciplinares, Zaragoza, vol. 5 : p. 165-199.

Keller G. and BarRera E. (1990): The Cretaceous/Tertiary boundary impact hypothesis and the paleontological record. Geol. Soc. Amer. Spec. Pap., Boulder, vol. 247, p. 563-575.

Keller G., Barrera E., Schmitz B. and Mattson E. (1993): Gradual mass extinction, species survivorship, and long-term environmental changes across the Cretaceous/Tertiary boundary in high latitudes. Geol. Soc. Amer. Bull., Boulder, vol. 105 , p. 979-997.

Keller G., Li L. and MaCleod N. (1995) : The Cretaceous/Tertiary boundary stratotype section at El Kef, Tunisia : how catastrophic was the mass extinction? Palaeogeogr. Palaeoclimatol. Palaeoecol., Amsterdam, vol. 119, p. 221-254. 
Keller G. and MACLEod N. (1994) : Reply to comment on "The Cretaceous-Tertiary boundary transition in the Antarctic Ocean and its global implications'. Mar. Micropaleontol., Amsterdam, vol. 24, p. 101-118.

LAMOLDA M.A., (1983) : Biostratigraphie du Maastrichtien basco-cantabrique; ses foraminifères planctoniques. Géol. Méditer. Marseille, vol. X(3-4), p. 121-126.

LAMOLDA M.A. (1990) : The Cretaceous-Tertiary boundary crisis at Zumaya (Northern Spain). Micropaleontological data. In E.G. Kauffman and O.H. Walliser (Editors). Extinction events in Earth History. Lecture Notes in Earth Sciences, Springer Verlag. Berlin, vol. 30, p. 393-399.

Lamolda M.A., Orue-EtXchebarria $X$. and Proto-Decima $F$. (1983) : The Gretaceous-Tertiary boundary in Sopelana (Biscay, Basque Country). Zitteliana. München, vol. 10, p. 663670 .

Li L. and KELLER G., in press : Environmental changes during the last $300 \mathrm{kyr}$ of the Maastrichtian at El Kef, Tunisia. Palaios, Tulsa.

LinaRes D. (1977) : Estudio de los foraminíferos planctónicos del Cretácico superior de las Cordilleras Béticas (sector central). Doct. Thesis. Univ. Málaga. 410 p.

LiNARES D. and MARTINEZ-GaLLEgo J., (1971): Observaciones sobre el tránsito Cretaceo-Paleogeno en el secton de Alamedilla (provincia de Granada). Cuad. Geol., Granada, vol. 2, p. 137-146.

LuU C. and OLSSON K. (1994) : On the origin of Danian normal perforate planktonic foraminifera from Hedbergella. Journ. Foram. Res., Washington, vol. 24(2), p. 61-74.

Luterbacher H.P. and Premoli-Silva I. (1964) : Biostratigrafia del limite Cretaceo-Terziario nell'Appennino Centrale. Riv. Ital. Paleont. Strat., Milano, vol. 70(1), p. 67-128.

MACLEOD N. (1993): The Maastrichtian-Danian radiation of triserial and biserial planktic foraminifera : Testing phylogenetic and adaptational hypotheses in the (micro) fossil record. Mar. Micropaleontol., Amsterdam, vol. 21, p. 47-100.

Macleod N. and Keller G. (1991a): Hiatus distribution and mass extinctions at the Cretaceous/Tertiary boundary. Geo$\log$, Boulder, vol. 19, p. 497-501.

MACLEOD N. and Keller G. (1991b): How complete are the K/T boundary sections? Geol. Soc. Am. Bull., Boulder, vol. 103, p. 1439-1457.

MACLEOD N. and KELLER G., (1994) : Comparative biogeographic analysis of planktic foraminiferal survivorship across the Cretaceous/Tertiary (K/T) boundary. Paleobiology, Ithaca, vol. 20(2), p. 143-177.

Molins E. (1994) : Aspectos epistemológicos y causas de la extinción. In E. Molina (Editor), Extinción y registro fósil. Extinction and the fossil record. SIUZ Cuadernos Interdisciplinares, Zaragoza, vol. 5, p. 11-30.

Molina E. (1995): Modelos y causas de extinción masiva. Interciencia, Caracas, vol. 20(2), p. 83-89.

OfFICER C.B. and DRAKE C.L. (1985): Terminal Cretaceous environmental events. Science, Washington, vol. 277, p. 1161-1167.

ORLE-ETXCHERARRIA X. (1983-1984) : Los foraminíferos planctónicos del Paleógeno del sinclinorio de Bizkaia (corte de Sopelana-Punta de la Galea). Kobie, Bilbao, vol. 13, p. 175249 , vol. 14 , p. $351-429$.

Orue-Etxchebarria X., Lamolda M.A. and Apellaniz E. (1984) : Los foraminíferos planctónicos del Paleoceno vizcaino y su bioestratigrafía. Rev. Esp. Micropal., Madrid, vol. 16, p. 59-74.
Orue-EtXchebarria X., Rocchia R., Pujalte V., Apellaniz E. and BACETA J.I. (1991) : Estudio preliminar del tránsito Cretácico/Terciario en la sección del monte Urko (Eibar, País Vasco). I Congr. Esp. Terc., Vic, p. 240-243.

Pardo A., Keller G. and Ortiz N. (1996) : Latest Maastrichtian and $\mathrm{K} / \mathrm{T}$ boundary foraminiferal turnover and environmental changes at Agost, Spain. In N. MacLeod and G. Keller (Editors), Biotic and environmental events across the Cretaceous/Tertiary boundary. Norton Press, New York. (in press).

Premoll-Silva I. (1977) : The earliest Tertiary Globigerina eugubina Zone : paleontological significance and geographical distribution. Mem. Seg. Cong. Latin. Geol., Caracas, vol. 3, p. 1541-1555.

Robaszynski F., Caron M., Gonzalez-Donoso J.M., Wonders A.H. and EwgPF (1983-1984): Atlas of late Cretaceous Globotruncanids. Rev. Micropaléont., Paris, vol. 36(3-4), p. $145-305$.

Sснміт٪ B. (1994) : Geochemical high-resolution stratigraphy of Cretaceous/Tertiary boundary in Denmark, Spain and New Zealand. In E, Molina (Editor), Extinción y registro fósil. Extinction and the fossil record. SIUZ Cuadernos Interdisciplinares, Zaragoza, vol. 5, p. 121-140.

Schmitz B., Keller G. and Stenvall O. (1992) : Stable isotope and foraminiferal changes across the Cretaceous-Tertiary boundary at Stevns Klint, Denmark : Arguments for long-term oceanic instability before and after bolide-impact event. $\mathrm{Pa}$ laeogeogr., Palaeoclimatol. Palaeoecol., Amsterdam, vol. 96, p. $233-260$.

SIGNOR P.W. and LIPPS J.H. (1982): Sampling bias, gradual extinction patterns and catastrophes in the fossil record. Geol. Soc. Am. Spec. Pap., Boulder, vol. 190, p. 291-296.

SмIт J. (1977) : Discovery of a planktonic foraminiferal association between the Abathomphalus mayaroensis Zone and the "Globigerina" eugubina Zone at the Cretaceous/Tertiary boundary in the Barranco del Gredero (Caravaca, SE Spain) : A preliminary report. Proc. Kon. Ned. Acad. Wet., Amsterdam, vol. 80(4), p. 280-301.

SMIT J. (1979) : The Cretaceous/Tertiary transition in the Barranco del Gredero, Spain. In W.K. Christensen and T. Birkelund (Editors), Proc. C-T bound. events Symp., vol. II, p. 156-163.

SMIT J. (1982) : Extinction and evolution of planktonic foraminifera after a major impact at the Cretaceous / Tertiary boundary. Geol. Soc. Amer. Spec. Paper, Boulder, vol. 190, p. 329-352.

Smiт J. (1990): Meteorite impact, extinctions and the Cretaceous-Tertiary Boundary. Geol. Mijnbouw, The Hague, vol. 69 , p. 187-204.

SMIT J. (1994) : Blind tests and muddy waters. Nature, London, vol. 368 , p. 809-810.

SMIT J. and HERTOGEN J. (1980) : An extraterrestrial event at the Cretaceous-Tertiary boundary. Nature, London, vol. 285, p. $198-200$.

SMIT J. and TEN KATE W.G.H.Z. (1982) : Trace element patterns at the Cretaceous-Tertiary boundary : consequences of a large impact. Cretaceous Res., London, vol. 3, p. 307-332.

Solakius N., Manmouri A.L. and Bensalem H. (1984) : Planktic foraminiferal biostratigraphy of the Maastrichtian sedimentary beds at Ain Mdeker, Northeastern Tunisia. Geobios, Lyon, vol. 17(5), p. 583-591.

Venkatesan T.R., Pande K. and Gopalan K. (1993) : Did Deccan volcanism pre-date the Cretaceous/Tertiary transition? Earth Plan. Sci. Lett., Amsterdam, vol. 119, p. 181189. 